\title{
A Panchromatic View of Brown Dwarf Aurorae
}

\author{
J. Sebastian Pineda ${ }^{1,2}$ (D), Gregg Hallinan ${ }^{2}$, and Melodie M. Kao ${ }^{2}$ (D) \\ ${ }^{1}$ University of Colorado Boulder, Laboratory for Atmospheric and Space Physics, 3665 Discovery Drive, Boulder CO, 80303, USA \\ ${ }^{2}$ California Institute of Technology, Department of Astronomy, 1200 E. California Avenue, Pasadena CA, 91125, USA \\ Received 2017 January 22; revised 2017 July 28; accepted 2017 August 8; published 2017 September 1
}

\begin{abstract}
Stellar coronal activity has been shown to persist into the low-mass star regime, down to late M-dwarf spectral types. However, there is now an accumulation of evidence suggesting that at the end of the main sequence, there is a transition in the nature of the magnetic activity from chromospheric and coronal to planet-like and auroral, from local impulsive heating via flares and MHD wave dissipation to energy dissipation from strong large-scale magnetospheric current systems. We examine this transition and the prevalence of auroral activity in brown dwarfs through a compilation of multiwavelength surveys of magnetic activity, including radio, X-ray, and optical. We compile the results of those surveys and place their conclusions in the context of auroral emission as a consequence of large-scale magnetospheric current systems that accelerate energetic electron beams and drive the particles to impact the cool atmospheric gas. We explore the different manifestations of auroral phenomena, like $\mathrm{H} \alpha$, in brown dwarf atmospheres and define their distinguishing characteristics. We conclude that large-amplitude photometric variability in the near-infrared is most likely a consequence of clouds in brown dwarf atmospheres, but that auroral activity may be responsible for long-lived stable surface features. We report a connection between auroral $\mathrm{H} \alpha$ emission and quiescent radio emission in electron cyclotron maser instability pulsing brown dwarfs, suggesting a potential underlying physical connection between quiescent and auroral emissions. We also discuss the electrodynamic engines powering brown dwarf aurorae and the possible role of satellites around these systems both to power the aurorae and seed the magnetosphere with plasma.
\end{abstract}

Key words: brown dwarfs - planets and satellites: aurorae - stars: activity

\section{Introduction}

Within the past 15 years, the discovery and follow-up observations of radio emission from brown dwarfs (e.g., Berger et al. 2001; Hallinan et al. 2007; Route \& Wolszczan 2012; Hallinan et al. 2015; Kao et al. 2016) have heralded a shift in our understanding of magnetic activity in low-mass stars and ultracool dwarfs (UCDs; spectral type $\geqslant$ M7). The accumulating evidence now suggest that there may be a transition at the end of the main sequence away from coronal/chromospheric solar-like magnetic activity toward auroral-planet-like phenomena, from current systems driven by local photospheric plasma motions to those driven by a global electrodynamic interaction in the large-scale magnetosphere. Moreover, the progress of several observational surveys of brown dwarfs across the electromagnetic spectrum allows us to put together a comprehensive view of UCD auroral phenomena, for the first time, in this article. However, in order to put these observations in context, it is essential to discuss both the standard coronal/ chromospheric picture of stellar magnetic activity and the underlying processes that govern aurorae in the gas-giant planets of the solar system.

\subsection{Stellar Activity in Low-mass Stars}

Our understanding of stellar magnetic activity is rooted in our understanding of the Sun. Solar observations of a host of phenomena, from impulsive flare events to long-term monitoring of sunspots as well as the study of coronal and chromospheric structures, have formed the basis for interpreting observations of similar activity in M-dwarfs (e.g., Haisch et al. 1991). Observations indicate that a version of the same mechanisms powering solar magnetic activity operates in low-mass stars. The process requires an internal dynamo that generates the persistent magnetic field anchored deep in the stellar interior and the non-thermal local heating of the upper atmosphere, above the photosphere, through magnetic reconnection and/or MHD wave dissipation (e.g., Linsky 1980).

In early M-dwarfs, with partially convective interiors, the dynamo is thought to be the same as that operating in the Sun, the $\alpha \Omega$ dynamo, which depends in part on the shearing layer between the radiative core and the convective envelope to transfer rotational energy into magnetic energy, linking the magnetic activity to the rotation and internal structure of the star (e.g., Ossendrijver 2003; Browning et al. 2006). This leads to strong feedback between a star's rotational evolution, due to angular momentum loss in a stellar wind, and observable tracers of magnetic phenomena (e.g., Covey et al. 2011; Reiners \& Mohanty 2012). For example, younger and more rapidly rotating $\mathrm{M}$-dwarfs flare more frequently than similar older stars, depositing energy in their upper atmospheres at a higher rate early in their lifetimes (Hilton et al. 2010). The connection is further observed as a strong correlation between the stellar rotation/age and emission lines that trace upper atmospheric heating (e.g., Skumanich 1972). Emission features such as $\mathrm{Ca} \mathrm{II} \mathrm{H}$ and $\mathrm{K}$, and $\mathrm{H} \alpha$ are more prevalent and stronger in faster rotating M-dwarfs compared to slower rotators (Delfosse et al. 1998; Mohanty \& Basri 2003; West et al. 2008, 2015; Browning et al. 2010).

Although these features for early M-dwarfs are indicative of chromospheric atmospheric structures, their decline in slowly rotating stars does not indicate the disappearance of inverted atmospheric temperature profiles. As indicated by observations at ultraviolet (UV) wavelengths, chromospheric, transition region, and coronal emission lines, such as $\mathrm{Mg}$ II at $2796 \AA$, N V at $1239 \AA$ and $1243 \AA$, and Fe XII at $1242 \AA$, are prevalent in M-dwarf atmospheres, even for slowly rotating M-dwarfs that 
are "inactive" in $\mathrm{H} \alpha$ (France et al. 2013, 2016). In some M-dwarfs, $\mathrm{H} \alpha$ in absorption may actually reflect weak chromospheric activity (Cram \& Mullan 1985). Indeed, many weakly active $\mathrm{M}$-dwarfs with $\mathrm{Ca}$ II $\mathrm{H}$ and $\mathrm{K}$ emission lines are known to display $\mathrm{H} \alpha$ absorption features (e.g., Walkowicz \& Hawley 2009). The presence of coronal structures, like those in the Sun, in M-dwarf atmospheres, is further corroborated by the detections of X-ray emission in observations of early M-dwarfs (e.g., James et al. 2000; Pizzolato et al. 2003). Like the optical emission features, UV and X-ray emission are also strongly correlated with rotation/age, with observations showing constant emission levels for young objects rotating more quickly than $\sim 5$ days, and the emission declining for more slowly rotating objects as they age (Pizzolato et al. 2003; Cook et al. 2014; Shkolnik \& Barman 2014).

Early M-dwarf radio emission also appears to be consistent with this coronal/chromospheric picture. From F-type dwarf stars to early M-type dwarf stars, the Güdel-Benz relation demonstrates a tight empirical relation between coronal X-ray and quiescent radio emission, illustrating a deep connection between the coronal plasma producing the X-ray emission and the non-thermal energetic electrons responsible for the radio emission (Guedel \& Benz 1993). The persistent heating of this coronal plasma to over $10^{6} \mathrm{~K}$ is typically associated with strong small-scale fields and their turbulent reconnection (e.g., Rosner et al. 1985; Solanki et al. 2006). Indeed, the results of Zeeman Doppler Imaging (ZDI) studies demonstrate that active flaring early M-dwarfs exhibit complicated non-axisymmetric multipolar large-scale fields similar to what is seen on the Sun, suggesting the presence of significant magnetic structures that heat and power the coronal radio and X-ray emission (e.g., Donati et al. 2008).

\subsection{Auroral Processes in Planetary Magnetospheres}

In contrast to the stellar paradigm, planetary auroral emissions are associated with large-scale field-aligned current systems that pervade the extended magnetosphere, connecting the planetary atmosphere to energetic processes in the middle magnetosphere. In the solar system, there are three main mechanisms that generate auroral currents (see Keiling et al. 2012 and references therein). First, the interaction between the solar wind and a planetary magnetosphere triggers magnetic reconnection events that accelerate electrons along the magnetic field lines. This mechanism dominates the aurorae of the Earth and Saturn (e.g., Cowley et al. 2004). Second, the relative motion of an orbiting satellite through a planet's magnetosphere creates a current system in the flux tube connecting the moon and the planet. This mechanism produces the auroral emission associated with the moons Io and Enceladus of Jupiter and Saturn, respectively (e.g., Saur et al. 2004). Lastly, the breakdown of co-rotation between a rotating plasma disk and the planetary magnetosphere can create a shearing layer that drives auroral currents. This is the mechanism that powers the main Jovian auroral oval (e.g., Cowley \& Bunce 2001). Moreover, the different mechanisms can overlap, as they do in the Jovian magnetosphere. Each of these electrodynamic engines generates strong-field-aligned currents that drive accelerated electron beams, the fundamental ingredient of auroral emission processes.

The acceleration of electrons creates an energetic nonthermal energy distribution and can lead to the onset of the electron cyclotron maser instability (ECMI). The necessary criteria are an energy distribution dominated by the nonthermal component and a cyclotron frequency larger than the local plasma frequency,

$$
\frac{\omega_{p e}^{2}}{\omega_{c e}^{2}}=\frac{4 \pi n_{e} m_{e} c^{2}}{B^{2}} \leqslant 1,
$$

where $n_{e}$ is the electron density, $B$ is the magnetic field strength, $m_{e}$ is the electron mass, and $c$ is the speed of light (see Treumann 2006). As the ratio in Equation (1) approaches unity, the maser becomes weaker and less efficient. However, under the conditions of a dilute plasma immersed in a strong magnetic field, energetic electrons become an efficient radiation source. The result is a strong coherent radio source, emitting near the local cyclotron frequency, that is highly circularly polarized and beamed into a thin $\left(\sim 1^{\circ}\right)$ conical sheet with large opening angles, nearly perpendicular to the magnetic field direction, $\gtrsim 80^{\circ}$ (Dulk 1985; Treumann 2006). ECM radio emission has been observed in the magnetized planets of the solar system, signaling the presence of non-thermal energetic electron distributions in the regions around the planetary magnetic poles, near the top of the atmosphere (e.g., Zarka 1998).

The energetic electron beams responsible for the radio emission precipitate into the atmosphere and generate a cascade of additional auroral emission processes (see Badman et al. 2015 and references therein). In Jupiter and Saturn, where the atmospheres are predominantly hydrogen, the collision of the energetic electrons with the atmospheric gas leads to the excitation and ionization of $\mathrm{H} / \mathrm{H}_{2}$ and subsequent emissions at UV and optical wavelengths, including Lyman and Balmer line emissions (Perry et al. 1999; Vasavada et al. 1999; Grodent et al. 2003; Gustin et al. 2013; Dyudina et al. 2016). The creation of ionized species in the Jovian and Kronian auroral regions also leads to significant ion chemistry within the atmosphere and the creation of the strongly emitting species $\mathrm{H}_{3}^{+}$ (e.g., Perry et al. 1999). In Jupiter, the ro-vibrational transitions of $\mathrm{H}_{3}^{+}$serve to effectively cool the atmosphere and regulate exospheric temperatures (Maillard \& Miller 2011). The deposition of energy from the electron beam into the atmosphere also leads to a significant thermal contribution to the auroral emissions between 7 and $14 \mu \mathrm{m}$ (Bhardwaj \& Gladstone 2000). X-ray emission has also been detected in the auroral polar regions, a consequence of charge-exchange reactions of highly ionized species such as oxygen and sulfur, likely created during ion precipitation in auroral currents (e.g., Gladstone et al. 2002; Hui et al. 2009).

These different multiwavelength auroral emission processes are the consequence of the energy dissipation from the electrodynamic engine operating in the planetary magnetosphere. In the Jovian system, the bulk of the energy, $\sim 85 \%$, goes into atmospheric heating and thermal radiation (Bhardwaj $\&$ Gladstone 2000). Most of the remaining $~ 15 \%$ emerges as part of the UV emission, with less than $\sim 1 \%$ of the energy going into optical aurorae (Bhardwaj \& Gladstone 2000). Additionally, the radio contribution only represents $\lesssim 0.1 \%$ of the total auroral energy, and the X-rays represent even less (Bhardwaj \& Gladstone 2000).

\subsection{Brown Dwarfs: Between Stars and Planets?}

The divide between stars and planets reflects the different natures of the atmospheres and physical properties of these 
objects. However, brown dwarfs, as objects that span this separation, constitute a regime in which there could be a transition from the planetary regime to the stellar one. Observationally, many of the the atmospheric properties of brown dwarfs, such as effective temperature, $T_{\text {eff }}$, overlap with those of, on the low-mass end, gas-giant planets, and, on the high-mass end, very low-mass stars (e.g., Burrows et al. 2001). Since brown dwarfs cool over time, with core temperatures insufficiently high for sustained hydrogen burning throughout their lifetimes, individual objects may display $T_{\text {eff }} \sim 2700 \mathrm{~K}$ at early ages, but much cooler $T_{\text {eff }} \sim 1000 \mathrm{~K}$ at later ages, depending on the brown dwarf mass (Burrows et al. 2001). This property makes it difficult to distinguish individual objects without mass/age measurements, and consequently, a field population of brown dwarfs may be composed of a mix of objects with different ages and masses despite having similar effective temperatures (e.g., Burrows et al. 2001).

Although it is possible that a distinct form of magnetic phenomenon is manifest in the brown dwarf regime, the similarities in atmospheric properties make it plausible that magnetic phenomena may also change continually across the brown dwarf regime from planets to stars. Indeed, the underlying magnetic dynamos of giant planets, brown dwarfs, and very low-mass stars might be very similar (Christensen et al. 2009; Morin et al. 2011); however, this idea is currently being tested (see Kao et al. 2016). Nevertheless, the nature of the transition in magnetic activity across the brown dwarf regime is an open question, as is its dependence on physical properties such as mass and age. With the discovery of cooler and lower-mass brown dwarfs, and new evidence pointing to a breakdown of the coronal/chromospheric solar-like paradigm of magnetic activity, we are further motivated to consider the activity of brown dwarfs from the auroral-planet perspective. Consequently, both stellar and planet perspectives can be used to elucidate the nature of brown dwarf magnetic processes. In Section 2, we discuss how the multiwavelength trends in magnetic activity shift in the UCD regime. In Section 3, we examine the activity data in the context of auroral phenomena in brown dwarf atmospheres. Lastly, in Section 4, we provide our conclusions, while we summarize our findings in Section 5.

\section{Trends in UCD Magnetic Activity}

The multiwavelength features of stellar magnetic activity change at the end of the main sequence, for late M-dwarfs and UCDs. The shift in observational features is a consequence of significant differences between the stellar and substellar regimes, reflecting changes in the internal structure, the largescale magnetic field topology, and the atmospheric fractional ionization.

\subsection{Convection, Dynamos, and Rotation}

The lower mass and luminosity of very low-mass stars and brown dwarfs, relative to earlier-type stars, have significant consequences for their internal structure. In contrast to early M-dwarfs, these objects have convective interiors extending from their cores through to their outer layers. Consequently, a distinct dynamo mechanism must operate in this fully convective regime (mass $\lesssim 0.3 \quad M_{\odot}$, spectral type $\gtrsim \mathrm{dM} 4$; Chabrier \& Baraffe 2000) in order to sustain the observed kilogauss magnetic field strengths of these objects (Reiners \& Basri 2007). One commonly invoked dynamo is the $\alpha^{2}$

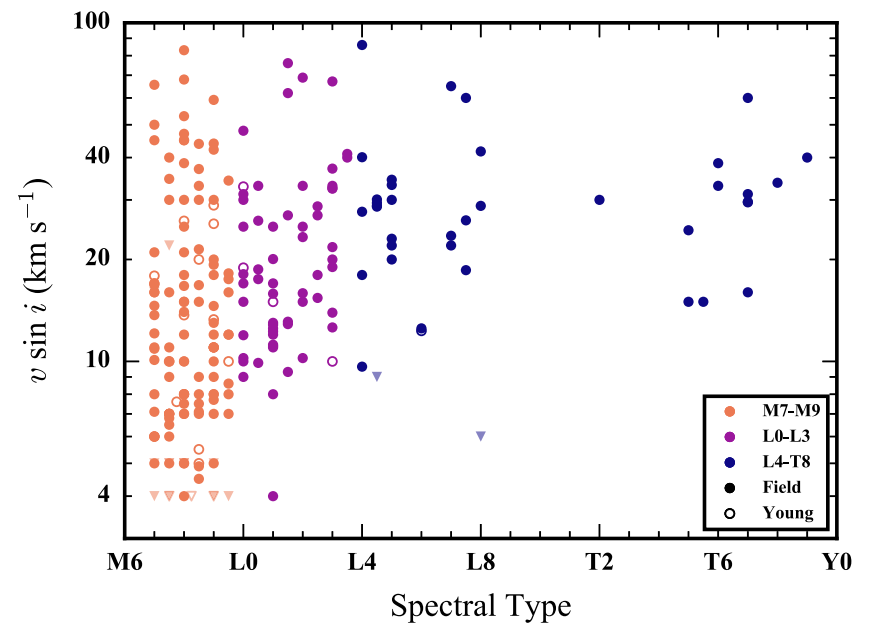

Figure 1. $v \sin i$ of UCDs as a function of spectral type, as compiled from the literature (see the Appendix). The brown dwarfs all show fast rotation rates and short periods, even at typical field ages. Circles are detections, with filled vs. open indicating field ages and indications of youth, respectively. Upper limits are plotted as triangles, with points grouped in different colors according to expected regimes of atmospheric ionization; see Section 2.2.

dynamo, which harnesses convective motions and rotation, but models have identified alternate dynamo mechanisms depending on a range of properties, including rotation rate and bolometric luminosity (Browning 2008; Christensen et al. 2009; Yadav et al. 2015). Interestingly, despite the transition in the internal structure, there does not appear to be an abrupt change in the strength of magnetic activity emission indicators across the fully convective boundary (e.g., West et al. 2015; Wright \& Drake 2016; Newton et al. 2017).

This transition coincides with a change in the prevalent magnetic field topologies. ZDI observations of fully convective M-dwarfs show the emergence of strong, large-scale, dipolar fields in mid M-dwarfs compared to the multipolar fields of earlier stars (Donati et al. 2006; Morin et al. 2010). This appears to persist into the UCD regime, where both kinds of field topologies have been observed, suggesting either a bi-stability of the dynamo mechanisms or potential phase transitions between dynamo modes (Morin et al. 2011; Kitchatinov et al. 2014). However, current ZDI observations are only available for objects of spectral type M9 or earlier, often limited by the faint luminosities and fast rotation rates of many brown dwarfs, necessitating an extrapolation of likely field topologies from warmer UCDs to cooler objects (see Kao et al. 2016).

This change in topology had been thought to potentially drive changes in the angular momentum evolution of mid-tolate M-dwarfs as seen in the observed distribution of rotation periods, $P$, and projected rotational velocities, $v \sin i$ (Irwin \& Bouvier 2009). However, Reiners \& Mohanty (2012) suggested that the rise in rotation rates of fully convective stars compared to earlier stars may be driven predominantly by changes in the stellar radius. The increase in the observed rotation rates of very low-mass stars extends throughout the UCD regime where the object radius is nearly independent of mass and is similar to the radius of Jupiter (e.g., Chabrier \& Baraffe 2000). In Figure 1, we plot a compilation of numerous literature sources (see the Appendix) of $v \sin i$ measurements, illustrating the large rotational velocities of UCDs and, correspondingly, their fast rotation rates $\left(v \sim 20 \mathrm{~km} \mathrm{~s}^{-1}\right.$ $\rightarrow P \sim 6.2 \mathrm{hr}$, for a radius of $\left.1 R_{\mathrm{Jup}}\right)$. Indeed, most UCDs show signs of short rotation periods, with many showing periods of 
only a couple of hours, sustaining these fast rotation rates even at field ages (Hallinan et al. 2008; Metchev et al. 2015). This further suggests that UCDs do not have strong stellar winds that remove angular momentum as they do in stars, likely a consequence of the largely neutral atmospheres and diminished coronal activity (see Section 2.2). Traditionally, the Rossby number, $R o=P / \tau_{c}$, where $\tau_{c}$ is the convective overturn timescale, has been used to quantify the effect of rotation on magnetic activity in stars; however, following Kiraga \& Stepien (2007) and Reiners \& Basri (2010), the convective overturn timescale is a constant in the UCD regime and may not even be well-defined for these objects. Consequently, we use the rotational velocity, as a broadly available observable, to compare the effects of rotation on magnetic phenomena among UCDs.

\subsection{Chromospheres and Coronae?}

The lower luminosities of very low-mass stars and brown dwarfs lead to much cooler $T_{\text {eff }}$, and correspondingly, to much less ionized atmospheres. Mohanty et al. (2002) used atmospheric models to show that atmospheres below $2300 \mathrm{~K}$ are insufficiently ionized to support atmospheric current systems that sustain chromospheric and coronal activities. Recently, Rodriguez-Barrera et al. (2015), taking a similar approach with more recent atmospheric models, suggested that the corresponding threshold is closer to a temperature of $1400 \mathrm{~K}$, with variations depending on the atmospheric gravity and metallicity. Moreover, they show that, in these objects, significant portions of the atmosphere can be dominated by electromagnetic interactions, since the typical cyclotron frequency (assuming kilogauss field strengths) far exceeds the collision frequency of electrons with neutrals (Rodriguez-Barrera et al. 2015).

These theoretical studies have important implications for the observed magnetic emissions of UCDs. We thus use these temperature thresholds as guides when considering the changes in the observed properties of UCDs with spectral type $\left(T_{\text {eff }}\right)$, grouping the M-dwarfs (M7-M9), early L-dwarfs (L0-L3), and late L-dwarfs and T-dwarfs (L4-T8) together (e.g., Figure 1). Although an effective temperature of $2300 \mathrm{~K}$ roughly coincides with the $M / L$ transition, we additionally base our groupings in spectral type on the observational findings of Pineda et al. (2016) and Miles-Páez et al. (2017) with regard to the prevalence of $\mathrm{H} \alpha$ emission across the UCD regime, which suggests that the break below which ionization is too low to sustain chromospheric activity is closer to L4, $T_{\text {eff }} \sim 1600 \mathrm{~K}$. We focus on $\mathrm{H} \alpha$ and radio emission in Sections 2.2.1 and 2.2.2, respectively, as the most extensively studied tracers of magnetic emissions in the UCD regime.

However, the changes in the ability of UCDs to sustain significant magnetic heating of their upper atmospheres relative to stars is also manifest in additional multiwavelength observations of magnetic phenomena. Photometric UV data on late M-dwarfs reveal predominantly weak NUV and FUV emissions (Jones \& West 2016), with spectroscopic data on a few targets showing transition region emission features in the FUV (Hawley \& Johns-Krull 2003). The UV emissions of even cooler objects, L-dwarfs and later, have remained largely unexplored. The UCD regime also exhibits a steep drop in $\mathrm{X}$-ray emission relative to earlier-type stars (e.g., Berger et al. 2010; McLean et al. 2012; Williams et al. 2014). In Figure 2, we plot the quiescent X-ray luminosity of UCDs as a function

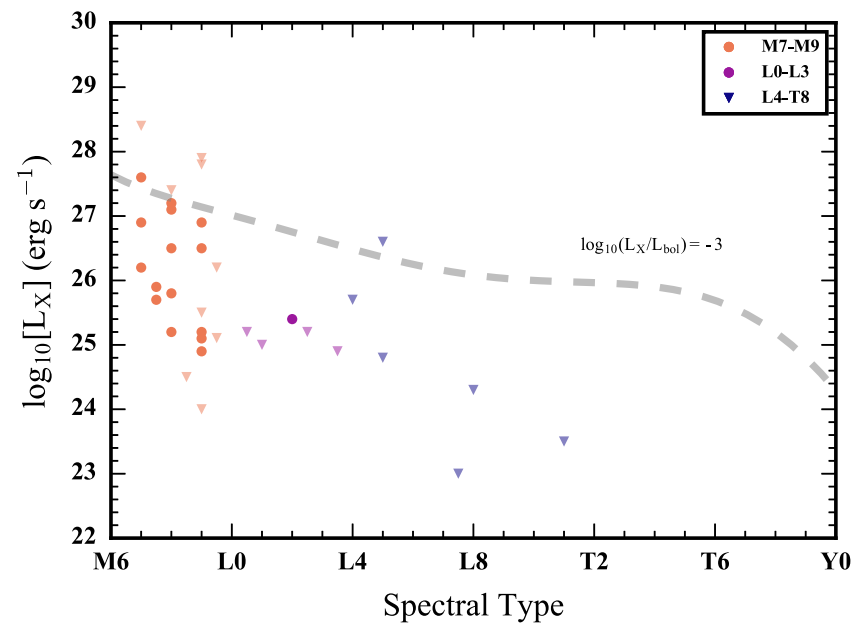

Figure 2. X-ray quiescent luminosities of UCDs as a function of spectral type as compiled from the literature (see the Appendix). Objects with X-ray detections typically also show flaring emission; see Williams et al. (2014). Relative to earlier-type stars, there is a steep drop-off in the observed X-ray emission for UCDs, with only one detection in objects later than L0. The dashed line indicates the typical X-ray emission level of active early M-dwarfs (Berger et al. 2010).

of spectral type as compiled in the literature (see the Appendix). Although some individual objects at the end of the main sequence are capable of heating a high-temperature corona, the overall lack of X-ray and UV detections, despite the fast rotation rates of the objects in these samples, points toward the diminishing ability of UCDs to sustain hot coronae.

Additionally, observations of flare events in UCD atmospheres suggest that the same processes generating flares in $\mathrm{M}$ stars continue to operate in some UCDs. These flare events are evident in the red optical data, noting the large increase in Balmer line emissions over short time intervals (e.g., Liebert et al. 1999; Schmidt et al. 2007). Monitoring of L-dwarfs with Kepler and concurrent spectroscopic observations revealed that these white light flares resemble the same kinds of events on earlier-type M-dwarfs (Gizis et al. 2013, 2017). The flares are just as energetic with energies as high as $\sim 10^{32}$ erg and potentially as strong as $\sim 10^{34}$ erg (Gizis et al. 2013; Schmidt et al. 2014a). Gizis et al. (2013) also demonstrated that energetic flares occur less frequently on their target L-dwarf by factors of 10-100 than what is observed on early M-dwarf flare stars. These observations may reflect the decreased ability of cooler UCD atmospheres to build up and release energetic flaring magnetic loops from buoyant flux tubes that have risen from the deep interior (Mohanty et al. 2002). Although some brown dwarfs are still able to generate these flares, as with the X-ray coronae and UV transition region emission lines, these data would suggest even fewer such flaring UCDs among the cooler late L-/dwarfs and T-dwarfs.

\subsection{1. $H \alpha$}

The $\mathrm{H} \alpha$ emission of UCDs also diverges from that observed in stellar atmospheres. For "active" early M-dwarfs, the strength of $\mathrm{H} \alpha$ emission is roughly in line with a normalized level of $\log _{10}\left(L_{\mathrm{H} \alpha} / L_{\mathrm{bol}}\right)=-3.8$ (Berger et al. 2010); however, the strength of emission in cooler objects is much weaker and declines more rapidly than the bolometric luminosity. In Figure 3, we show this decline by plotting the observed $\mathrm{H} \alpha$ luminosity as a function of spectral type in the UCD regime 


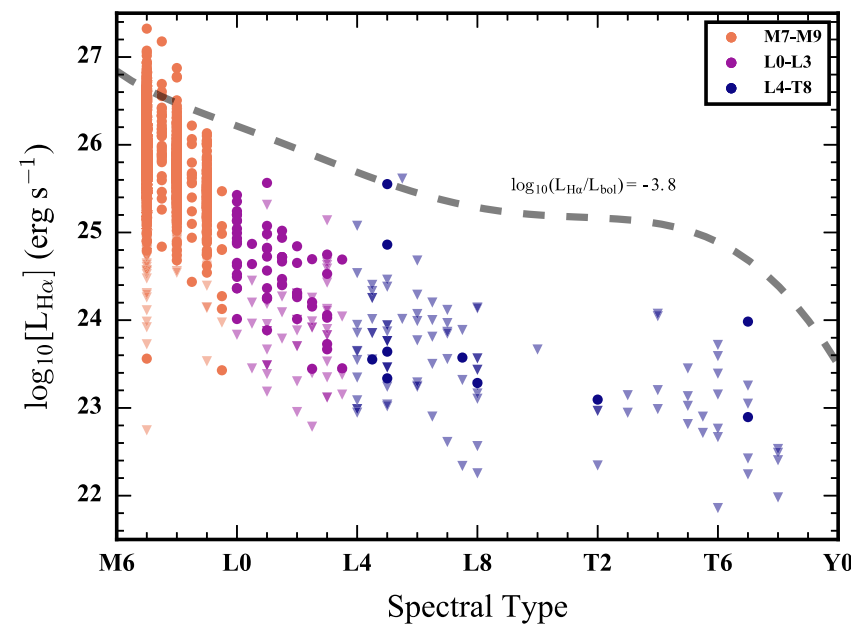

Figure 3. Luminosity in $\mathrm{H} \alpha$ in the UCD regime as a function of spectral type, spanning M7-T8, illustrating the decline in emission strength with effective temperature and the deviation from typical stellar emission strengths. Detections are shown as filled circles and non-detections as triangles. We use the polynomial relations of Filippazzo et al. (2015) to determine the bolometric luminosity, $L_{\mathrm{bol}}$, as a function of spectral type. The different UCDs are further grouped into different colors according to the expected regimes of atmospheric ionization; see Section 2.2. The data have been compiled from the literature (see the Appendix).

with a dashed line indicating a constant level of $L_{\mathrm{H} \alpha} / L_{\mathrm{bol}}$ for early M-dwarfs. The typical emission level departs considerably from the expected chromospheric value based on the bolometric luminosity.

This decline appears to be more gradual than what is observed in X-rays (see Figure 2), where the drop is more dramatic for L-dwarfs and cooler objects. In Figure 4, we plot each object's X-ray emission against their $\mathrm{H} \alpha$ emission, both normalized by their bolometric luminosities. For M7-M9 objects, there is a clear correlation between the observed $\mathrm{H} \alpha$ and X-ray luminosities. The best-fit line for these points is given as

$$
\log _{10}\left(L_{X} / L_{\mathrm{bol}}\right)=1.65 \log _{10}\left(L_{\mathrm{H} \alpha} / L_{\mathrm{bol}}\right)+2.86,
$$

with a 0.39 dex scatter on the relation at fixed $\mathrm{H} \alpha$ luminosity. Although we use typical values for these observed quantities, since the optical and X-ray observations are taken at different times, this scatter may be due predominantly to the intrinsic variability of the emission processes. Nevertheless, the correlation shows a clear connection between coronal and chromospheric heating processes in the warmest UCDs, which does not appear to persist into the coolest objects, although more data are needed. If M7-M9 dwarfs behave like warmer stars, there is a limit to extrapolating this relation to high energies as the emission likely saturates, $\log _{10}\left(L_{X} / L_{\text {bol }}\right) \sim-3$ (e.g., Pizzolato et al. 2003).

To better understand these trends in $\mathrm{H} \alpha$ as a function of spectral type, we have constructed the empirical cumulative distribution functions (ECDFs) for this emission in UCDs and nearby mid M-dwarfs (from the Sloan Digital Sky Survey DR7 spectroscopic sample of West et al. 2011) using the KaplanMeier estimator, which takes into account non-detections. Equivalent width measurements consistent with $0 \AA$ or indicative of absorption are treated as non-detections. In weakly active objects, the emergence of a chromosphere initially manifests as stronger $\mathrm{H} \alpha$ absorption before the line is filled in

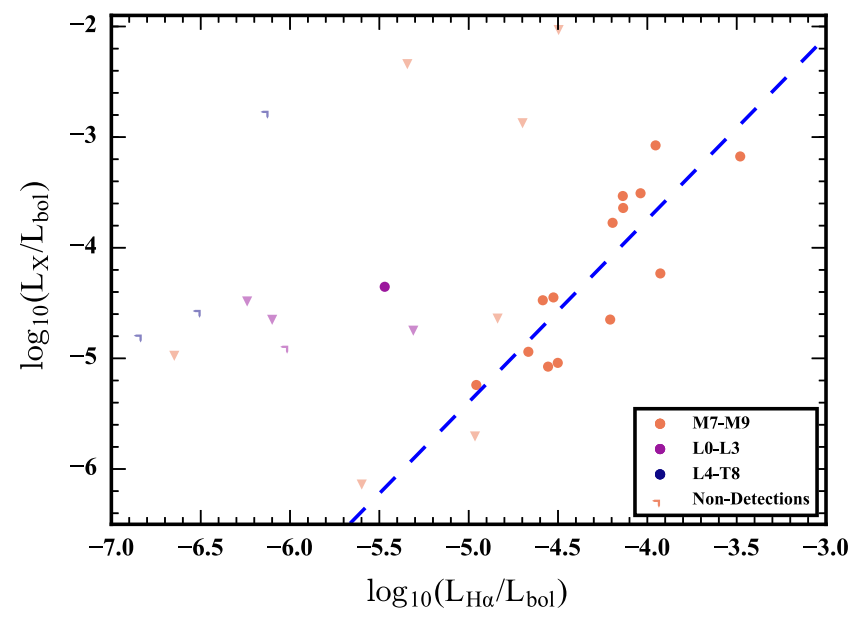

Figure 4. Normalized X-ray luminosity as a function of $\mathrm{H} \alpha$ luminosity in the UCD regime, showing all objects with measurements at both wavelength regimes in the literature (see the Appendix). Among M7-M9 dwarfs, there is a clear correlation between X-ray and $\mathrm{H} \alpha$ emissions. The dashed line represents the best-fit line for these points of slope 1.65 and intercept of 2.86 , with a scatter of 0.39 dex (see Equation (2)).

by stronger emission (Cram \& Mullan 1985), although for the coolest M-dwarfs the maximum observed absorption has equivalent width $\sim 0.075 \AA$ (Newton et al. 2017). Consequently, in this analysis, some very weakly active objects are treated as non-detections. Although some observations may not have been sufficiently sensitive to detect very weak emissions, the majority of the data set consists of observations that probed deep enough to detect typical emission levels (see Schmidt et al. 2015; Pineda et al. 2016). Furthermore, the Kaplan-Meier estimator provides a robust statistical treatment of the nondetections that permits a comparison of the ECDFs. In Figure 5, we plot the ECDFs of $L_{\mathrm{H} \alpha} / L_{\text {bol }}$ for objects with spectral types later than M4. The trend of cooler objects showing weaker emission is evident in how the curves shift to the left for later spectral-type objects. Where each curve meets the ordinate axis indicates the fraction of $\mathrm{H} \alpha$ non-detections in each spectral-type bin. Clearly, the cooler objects are less frequently observed in emission. The M5-M9 objects show similar rates of $\mathrm{H} \alpha$ detection, a consequence of restricting these bins to a height above the Galactic midplane, $|Z|<100 \mathrm{pc}$, where the typical ages are below the activity lifetimes of these stars (West et al. 2008). When including more distant objects, the mid M-dwarfs show fewer "active" stars, and hence, lower activity fractions (West et al. 2008; Pineda et al. 2013).

Because the ECDFs are constructed from all of the available literature, including many targets with only a single $\mathrm{H} \alpha$ observation, the distributions include scatter associated with the intrinsic stochastic variability of UCD $\mathrm{H} \alpha$ emission, as each individual object might have been in different activity states at the time of their respective observations. Consequently, the $\mathrm{H} \alpha$ variability, which can change by factors of 1.2-4 on short timescales (Lee et al. 2010), does not dramatically impact our comparison of the ECDFs across the UCD regime, especially for earlier-type objects with larger sample sizes. The uncertainty in these distributions is captured by the shaded regions in Figure 5, and we thus treat these distributions as representative of their respective populations.

In Figure 5, when comparing the cool brown dwarfs relative to the M-dwarfs, there is a stark change in the shape of the $\mathrm{H} \alpha$ distributions. Although the shapes are similar among the 


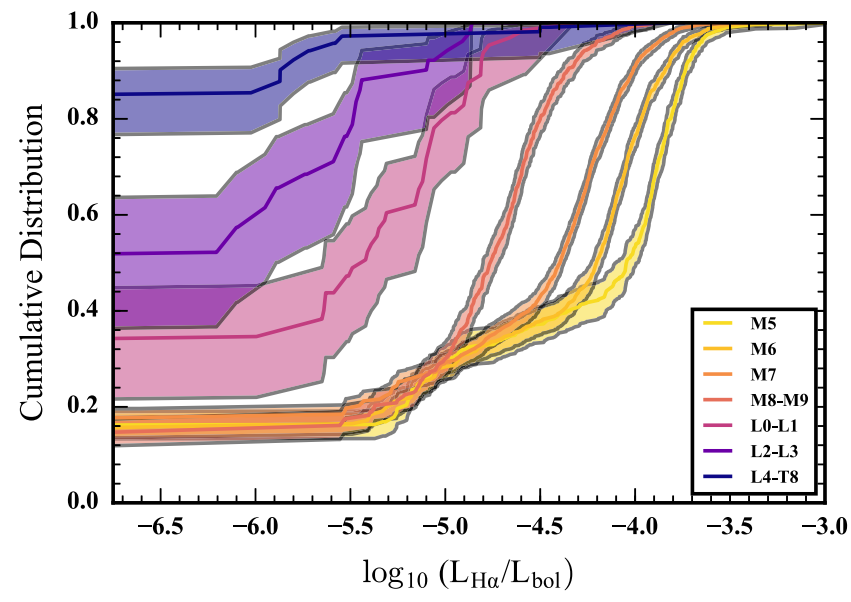

Figure 5. Empirical cumulative distribution functions of $\mathrm{H} \alpha$ luminosity normalized by the bolometric luminosity for late M-dwarfs and UCDs. Constructed using the Kaplan-Meier estimator, to account for non-detections, the curves illustrate the decline in the number of objects observed to be $\mathrm{H} \alpha$ active and the declining strength of the emission (also see Figure 3). The shaded regions represent $95 \%$ confidence intervals. The shape of the distributions are also distinct going from low-mass stars to cool brown dwarfs; see Section 2.2.1.

M-dwarfs, the cooler brown dwarfs are observed with $\mathrm{H} \alpha$ emission much less frequently, despite their rapid rotation (see Figure 1). Moreover, observations explicitly show that in the UCD regime, the $\mathrm{H} \alpha$ emission is not strongly correlated with the rotation distribution (e.g., Reiners \& Basri 2008; McLean et al. 2012, and references therein). In Figure 6, we show the distribution of UCDs with both $\mathrm{H} \alpha$ measurements and $v \sin i$ measurements in the literature (see the Appendix). There is no clear pattern of larger luminosities for faster rotators as has been observed in M-dwarfs (e.g., McLean et al. 2012; West et al. 2015).

The change in the $\mathrm{H} \alpha$ ECDFs for UCDs is likely largely attributable to the effects of atmospheric ionization. Cooler objects magnetically heat their atmospheres less efficiently despite maintaining large rotational velocities, leading to distributions that show both weaker and less prevalent $\mathrm{H} \alpha$ emission. Schmidt et al. (2015) further determined that this decline in activity coincided with a decreasing covering fraction for $\mathrm{H} \alpha$-emitting regions in L-dwarf atmospheres, which ostensibly vanish going into the T-dwarf regime. However, this chromospheric picture does not explain the distribution for the coolest brown dwarfs in Figure 5. Despite having atmospheres that are too cool to sustain much ionization, there are still a few objects that have strong $\mathrm{H} \alpha$ emission (Burgasser et al. 2003; Pineda et al. 2016). This manifests in the ECDF for the L4-T8 bin extending to values of $\log _{10}\left(L_{\mathrm{H} \alpha} / L_{\mathrm{bol}}\right)$ that are typical of late M-dwarfs (see Figure 5); consequently, the shape of the distribution for L4-T8 dwarfs does not fit neatly in the sequence defined by the predominantly chromospheric late M-dwarfs and early L-dwarfs. Although the difference in shape is driven by the strong emission of a few objects, like 2MASS J1237+6526, the existence of these extreme outliers points toward potentially distinct behavior in L4-T8 dwarfs. Whereas the bolometric luminosity declines throughout the L4-T8 bin, unlike for earlier spectral types, typical emission levels remain relatively constant across the L4-T8 range (see Figure 3). These data indicate that the $\mathrm{H} \alpha$ emission of the coolest brown dwarfs may be independent of $L_{\mathrm{bol}}$ and hence $T_{\mathrm{eff}}$, unlike what is observed in stars with strong evidence for chromospheres/coronae.

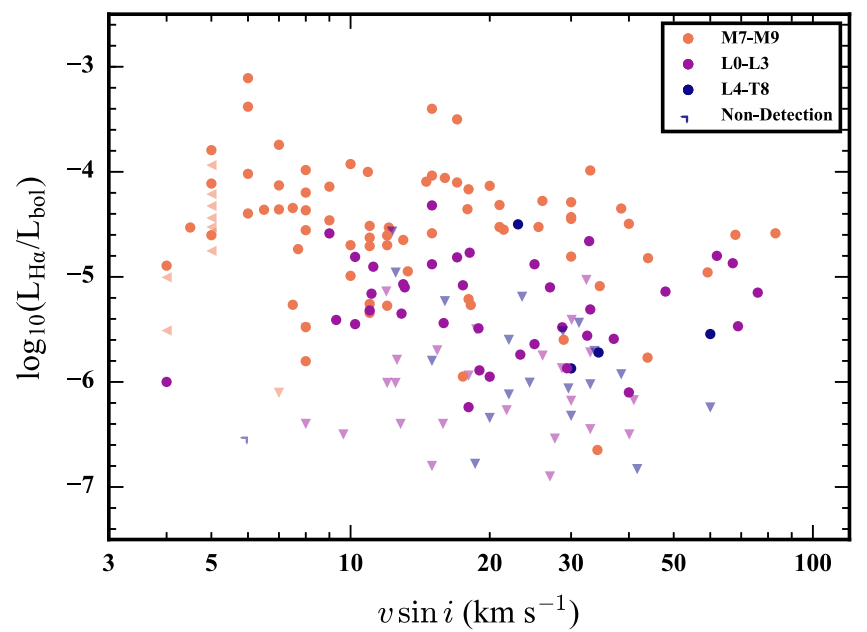

Figure 6. $\mathrm{H} \alpha$ emission of UCDs normalized by their bolometric luminosity as a function of projected rotational velocity. For UCDs, there does not appear to be a strong connection between $\mathrm{H} \alpha$ emission and rotation. The data have been compiled from the literature (see the Appendix).

These results suggest that a different mechanism is responsible for the $\mathrm{H} \alpha$ activity in these coldest brown dwarfs, distinct from the chromospheric emission seen in stars. The activity distribution across the UCD regime can thus be characterized as being predominantly chromospheric for late M-dwarfs, transitory across the early L-dwarf sequence, and not chromospheric for late L-dwarfs and T-dwarfs.

\subsubsection{Radio}

Unexpectedly, UCDs have also been observed to exhibit strong radio emission (Berger et al. 2001). Since the initial discovery, numerous surveys have looked for radio emission in very low-mass stars and brown dwarfs with very few detections (e.g., Berger 2006; McLean et al. 2012; Antonova et al. 2013). In Figure 7, we plot a compilation of radio observations of UCDs in the literature (see the Appendix) as a function of spectral type, showing only the quiescent radio luminosities in erg s ${ }^{-1} \mathrm{~Hz}^{-1}$, with observations typically conducted between $4 \mathrm{GHz}$ and $9 \mathrm{GHz}$. As discussed in Route \& Wolszczan (2016b), there appears to be a general decline in the strength of the emission with spectral type, despite a large scatter in the observed luminosities. The overall detection rate of unbiased radio surveys is $\sim 7 \%-10 \%$ (Route \& Wolszczan 2016b). In Figure 8, we plot the measured detection fraction as a function of $v \sin i$ for all UCDs with both radio observations and $v \sin i$ measurements. ${ }^{3}$ This plot shows an increase in the observed number of radio emission detections for quickly rotating objects, expanding on the results illustrated by McLean et al. (2012). Although fast rotators can be disguised with slow rotational broadening due to high inclinations, the faster rotators could be rotating even more quickly. Interestingly, we see a sharp rise in the detection fraction at a $v \sin i$ of $\sim 40 \mathrm{~km} \mathrm{~s}^{-1}$, which, for objects with radii of $1 R_{\text {Jup }}$ and inclination close to $90^{\circ}$, corresponds to a period of $3.1 \mathrm{hr}$. Although there may be an observational bias toward the detection of radio bursts (see below) in objects with rotation

\footnotetext{
3 We used the Adaptive Kernel Density Estimation routine akj within the quantreg package in $\mathrm{R}$ to construct probability density functions (PDF) for detections and non-detections as a function of $v \sin i$ and combined them to construct the detection fraction (Koenker 2016; R Core Team 2016).
} 


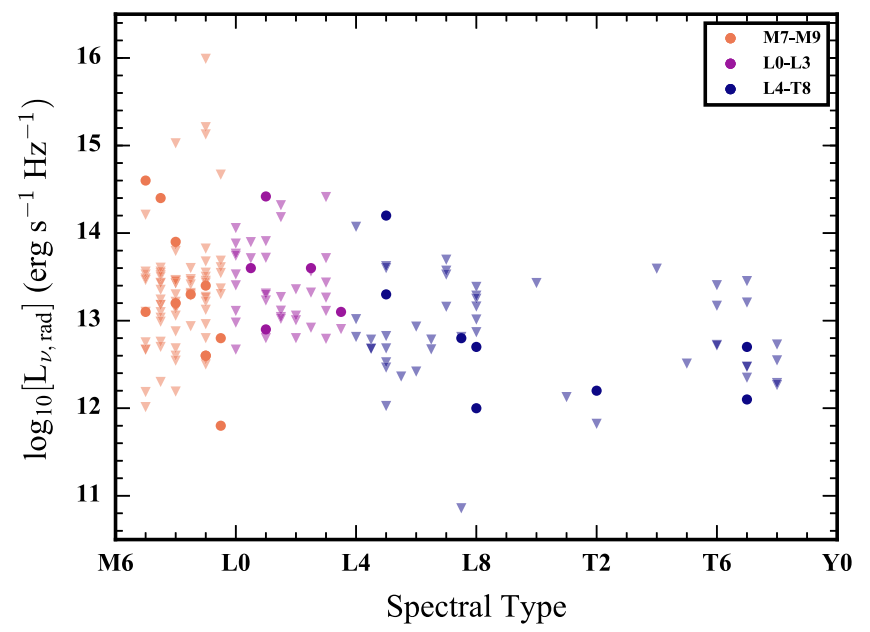

Figure 7. Radio luminosity of UCDs as a function of spectral type, showing significant radio sources even for the coolest brown dwarfs. Triangles denote upper limits and filled circles correspond to detections. The plotted points only indicate the quiescent emission levels, with several objects also displaying strong radio bursts; see Tables 1 and 2 . The data are compiled from the literature (see the Appendix). The different UCDs are grouped in different colors according to expected regimes of atmospheric ionization; see Section 2.2

periods less than the typical radio observing duration limits (usually several hours), this does not necessarily preclude the detection of the quiescent radio emission (as plotted in Figure 7 ); consequently, the trend evident in Figure 8 represents a real rise in the radio detection rates for faster rotators.

Significantly, the detected radio emission from these objects is morphologically distinct from that typically observed in lowmass stars. Quiescent stellar radio emission is likely dominated by a slowly varying gyrosynchrotron component at $\mathrm{GHz}$ frequencies, whereas stellar radio flares are highly energetic sporadic, impulsive events (see Güdel 2002 and references therein). Observations of UCDs have shown short-duration strong radio bursts at $\mathrm{GHz}$ frequencies superimposed on a quiescent background (e.g., Route \& Wolszczan 2012; Burgasser et al. 2015c). However, studies that have observed individual UCDs, from M8.5-T6.5, for extended periods, have often discovered the bursts to be periodic, highly circularly polarized, and have high brightness temperatures, indicative of coherent emission (e.g., Hallinan et al. 2007; Berger et al. 2009; Williams \& Berger 2015). Although the radio light curves show varying morphologies, the pulses are consistently periodic and in agreement with the ECMI. These radio emission properties provide direct evidence for the presence of stable auroral current systems, similar to those found in the solar system giant planets (see Section 1.2). We explore the aurora and its consequences further in Section 3. Although the acceleration mechanism is unclear, the quiescent radio components of UCDs appear consistent with gyrosynchrotron or synchrotron emission (Williams et al. 2015b).

The observations of pulsed ECMI radio emission throughout the UCD regime indicates the ability of these atmospheres to host conditions (see Equation (1)) amenable to the production of ECM radio sources, independent of the nature of UCD chromospheres and coronae (see below). The observed rate of periodically pulsing radio brown dwarfs is likely influenced by the properties of the ECM radio beaming. Interestingly, objects that exhibit a quiescent radio component have also shown strong periodic radio pulses when monitored for times exceeding their

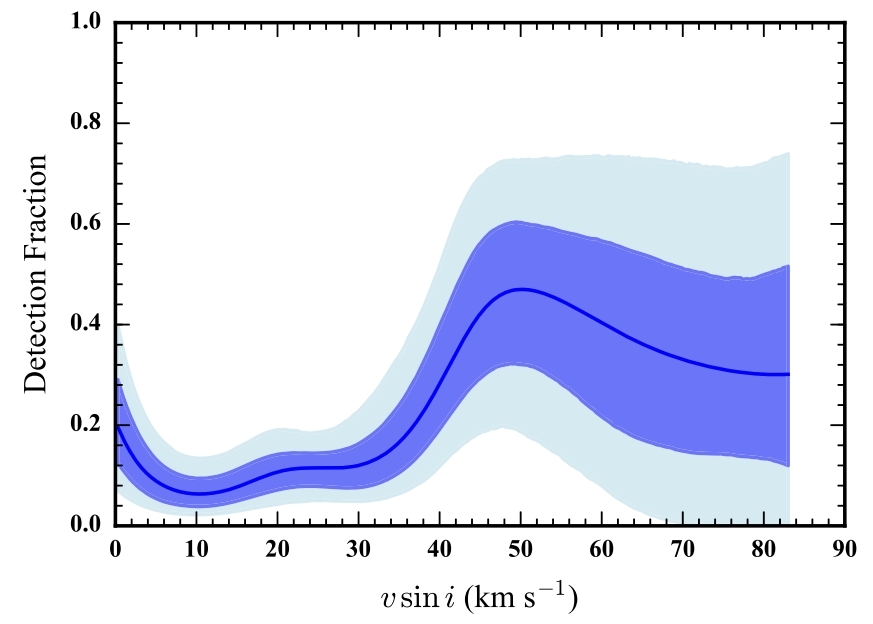

Figure 8. Detection fraction of UCDs, M7-T8, in the radio as a function of $v \sin i$, illustrating a rise in radio detections for faster rotating objects. The fraction is computed by comparing the radio detections to the non-detections using a non-parametric adaptive kernel density estimation. The dark blue shaded region denotes the $68 \%$ confidence interval, while the light blue region denotes the $95 \%$ confidence interval using 5000 bootstrap samples of the set of targets with radio observations and $v \sin i$ measurements.

rotational periods. For example, initial radio detections in short observations for TVLM 513-46546, LSRJ 1835+3259, 2MASS J0036+1821, 2MASS J1047+2124, and NLTT 33370 were followed up for several hours in extended monitoring that found periodic radio pulses (Hallinan et al. 2007, 2008; McLean et al. 2011; Route \& Wolszczan 2012). These results suggest that perhaps the pulses and the quiescent emission could be a consequence of the same underlying conditions. However, monitoring of other targets, such as DENIS J1048.0-3956, LP 944-20, and 2MASS J0952219-192431 did not detect periodic pulses, although this could be due to intrinsic variability, long rotational periods, and/or the effects of ECMI radio beaming (McLean et al. 2012; Lynch et al. 2016).

Pulsed radio emission is distinct from typical stellar behavior and is related to the underlying mechanisms that generate the ECMI. Moreover, additional clues can be gathered from a comparison to other wavelength bands. We noted the low detection rate of radio UCDs; however, that rate is bolstered by the success of recent studies using a targeted sampled, based on $\mathrm{H} \alpha$ emission in late L-dwarfs and T-dwarfs, suggesting a link between these emissions in the coldest brown dwarfs (Kao et al. 2016). We compare these two samples in Figure 9, showing the luminosity in $\mathrm{H} \alpha$ versus the radio luminosity, as in Figures 3 and 7, respectively. For the majority of late Mdwarfs, no radio emission is observed even when $\mathrm{H} \alpha$ emission is present. Although these objects often show variable $\mathrm{H} \alpha$ emission, the luminosities typically change by factors of only a couple (Lee et al. 2010). However, for L4-T8 objects, there is a significant overlap between objects showing both $\mathrm{H} \alpha$ and radio emissions (also see Tables 1 and 2).

The changes in the UCD radio behavior are also evident when comparing these observations to the X-ray observations, both in quiescence, as shown in Figure 10, which shows the GüdelBenz relation as a shaded stripe (Guedel \& Benz 1993; Benz \& Guedel 1994; Berger et al. 2010; Williams et al. 2014). The low X-ray luminosities (see also Figure 2) suggest only weak radio emissions, based on the coronal/chromospheric perspective; however, the observed quiescent radio luminosities are much 


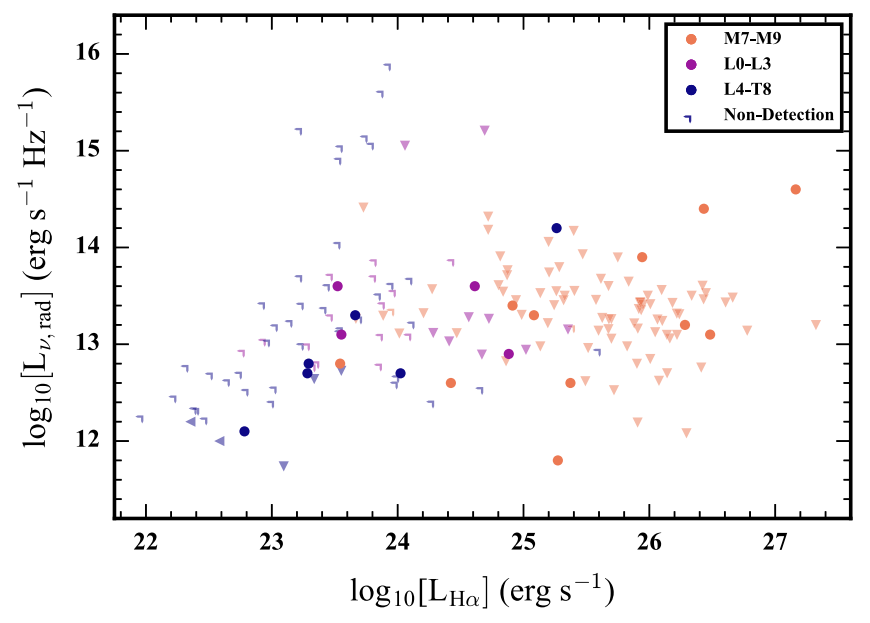

Figure 9. Radio luminosity plotted against the $\mathrm{H} \alpha$ luminosity in the UCD regime; no clear correlations are evident for all of the data. Triangles denote upper limits and filled circles correspond to detections. Non-detections at both wavelengths are shown as corner symbols. The data are compiled from the literature (see the Appendix), with the radio luminosity corresponding to quiescent emission levels as in Figure 7. The different UCDs are grouped into different colors according to expected regimes of atmospheric ionization; see Section 2.2

stronger, indicating that these processes might no longer be coupled in objects with these effective temperatures, $T_{\text {eff }} \lesssim 2600$ $\mathrm{K}$. Although these emissions might still be connected in some of the late M-dwarfs, consistent with the portion of objects displaying $\mathrm{H} \alpha$ chromospheric emission (see Section 2.2.1, Figure 4), the regime of magnetic activity as reflected by the radio emission is distinct. While the conditions capable of generating the ECMI and subsequent radio emissions are present, the conditions to generate substantial X-ray emission have vanished. Interestingly, some objects do show X-ray emission, and if it is attributable to weak coronal plasmas, the observed radio emission carries several orders of magnitude more energy than any associated synchrotron emission, as predicted by the Güdel-Benz relation. Considering both the $\mathrm{H} \alpha-$ X-ray relation (see Figure 4) and the Güdel-Benz relation, there are several objects that are consistent with the former but depart from the latter. If the radio is indeed decoupled from the X-ray for these objects, then these data could be explained by overlapping mechanisms in this regime, both coronal/chromospheric and ECMI related.

\subsection{Photometric Variability}

Traditional stellar photometric variability has been interpreted as evidence for starspots. However, as the atmosphere cools and becomes more neutral, the ability of the atmosphere to sustain these magnetic features becomes less clear. Concurrently, the low temperature of the atmospheres allows for the formation of dust condensates and clouds that influence the emergent stellar flux (e.g., Marley \& Robinson 2015). Thus, in UCD atmospheres, there is some ambiguity with regard to the dominant processes generating photometric variability, especially at the $M /$ $L$ transition, whether magnetic spots or clouds (Gizis et al. 2015). Doppler imaging of some example late M-dwarfs by Barnes et al. (2015) revealed high-latitude features that they interpreted as magnetic spots. However, these kinds of highlatitude features, and the photometric variability they generate,

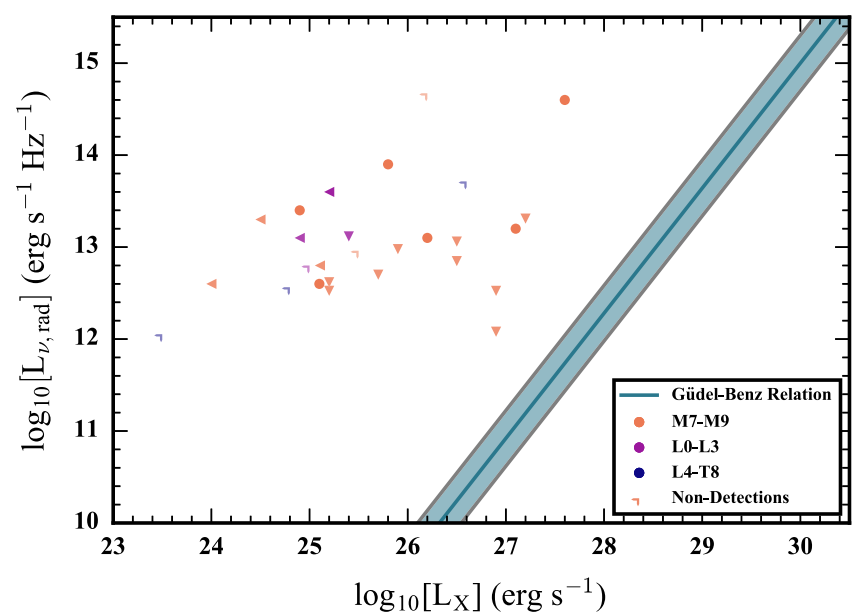

Figure 10. Quiescent radio luminosity vs. quiescent X-ray luminosity of UCDs for objects observed at both wavebands. The shaded strip shows the GüdelBenz relation and scatter for the tight X-ray-radio correlation in stellar coronal activity (Guedel \& Benz 1993; Williams et al. 2014). The radio-emitting UCDs strongly diverge from this empirical relation.

can also be interpreted as a consequence of an auroral electron beam (see Section 3.3.2).

Interestingly, photometric and spectroscopic monitoring of UCDs has revealed multiwavelength broadband variability across the full range of spectral types from late M-dwarfs to T-dwarfs (e.g., Apai et al. 2013; Harding et al. 2013a). Many of these observations have taken place in near-IR wavebands, predominantly $J$ and $K$, with monitoring observations of several hours. The results of these studies have revealed several large-amplitude variables located at the transition between L-dwarfs and T-dwarfs and some additional lower amplitude and less frequent variability in spectral types away from this transition (Radigan et al. 2012, 2014). These observations have been interpreted as evidence for a patchy transition in cloud cover from L-dwarfs to T-dwarfs (e.g., Artigau et al. 2009; Buenzli et al. 2014). Additionally, Spitzer monitoring of UCDs indicates that low-level variability is ubiquitous in the 3.6 and $4.5 \mu \mathrm{m}$ bands, also interpreted as clouds (Metchev et al. 2015). Many of the IR-variable objects also appear to display significant optical variability, with amplitudes of $\sim 10 \%$, comparable to the highest amplitude IR variables (Biller et al. 2013; Heinze et al. 2015).

The connection between optical and infrared variability is potentially significant in the context of magnetic activity because brown dwarfs with confirmed ECM radio emission also appear to show very clear long-term photometric variability at optical wavelengths (Harding et al. 2013a). Moreover, Hallinan et al. (2015) demonstrated that radio and optical variability may be linked as a consequence of auroral phenomena, distinct from standard starspot features (see Section 3.3.2).

Although it is possible that many of these processes are connected, both in optical and infrared, it is also probable that UCDs might display multiple sets of phenomena in different objects. One potentially distinguishing feature is the nature of the photometric variability. While much of the variability is seen to evolve with time, leading to irregular periodicity in extended monitoring, other observations show long-lived steady structures (Harding et al. 2013a; Crossfield et al. 2014; Buenzli et al. 2015; Gizis et al. 2015; Metchev et al. 2015). This difference may define a distinction between 
Table 1

Ultracool Dwarfs with Rotationally Periodic Radio Pulses

\begin{tabular}{|c|c|c|c|c|c|c|c|c|c|}
\hline Object & $\mathrm{SpT}$ & $\begin{array}{l}T_{\text {eff }} \\
(\mathrm{K})\end{array}$ & $\begin{array}{c}{\left[L_{\mathrm{bol}}\right]} \\
\left(L_{\odot}\right)\end{array}$ & $\begin{array}{l}\text { Mass } \\
\left(M_{\text {Jup }}\right)\end{array}$ & $\begin{array}{l}\text { Period } \\
(\mathrm{hr})^{\mathrm{a}}\end{array}$ & $\left(\mathrm{erg} \mathrm{s}^{-{ }^{-1}} \mathrm{~Hz}^{-1}\right)^{\mathrm{b}}$ & {$\left[L_{\mathrm{H} \alpha} / L_{\mathrm{bol}}\right]^{\mathrm{c}}$} & Phot. Var. ${ }^{\mathrm{d}}$ & References $^{\mathrm{e}}$ \\
\hline \multirow[t]{2}{*}{ NLTT $33370^{\text {f }}$} & M7 & $2954 \pm 3$ & -2.616 & $92.8 \pm 0.6$ & $\cdots$ & $<12.89$ & $\sim-3.8$ & MEarth, $g, i$ & $8,8, \mathbf{1 0}, \underline{\mathbf{1 9}}, \underline{\mathbf{2 6}}$ \\
\hline & M7 & $2947 \pm 4$ & -2.631 & $91.7 \pm 1.0$ & 3.89 & $14.6,(\mathbf{1 5 . 4})$ & $\cdots$ & $\ldots$ & \\
\hline LSR J1835+32 & M8.5 & $2316 \pm 51$ & -3.50 & $77 \pm 10$ & 2.84 & 13.3, (14.0) & $\sim-5.0$ & $I, R$ & $23,9, \mathbf{1}, \mathbf{1 2}, \underline{13}, 24$ \\
\hline TVLM 513-46 & M8.5 & $2242 \pm 55$ & -3.57 & $75 \pm 11$ & $1.9596^{\mathrm{h}}$ & $13.4,(\mathbf{1 4 . 8})$ & $\sim-5.1$ & $I, i^{\prime}, z^{\prime}, J$ & $16,9,5,7, \mathbf{1 1}, \overline{13}, \mathbf{2 0}, 27$ \\
\hline \multirow[t]{2}{*}{$\mathrm{J} 0746+2000^{\mathrm{g} \mathrm{i}}$} & L0.5 & $2205 \pm 50$ & -3.64 & $81.7 \pm 4.2$ & 3.32 & $\ldots$ & $\sim-5.2$ & I & $3,14, \overline{1} 8, \underline{\mathbf{2}}, \overline{13}, \mathbf{1 8}$ \\
\hline & L1.5 & $2060 \pm 70$ & -3.77 & $76.5 \pm 4.2$ & 2.072 & 13.6, (15.3) & $\ldots$ & $\ldots$ & \\
\hline $\mathrm{J} 0036+1821^{\mathrm{g}}$ & L3.5 & $1869 \pm 64$ & -3.93 & $66 \pm 13$ & 3.08 & $13.1,(\mathbf{1 3 . 8})$ & $\sim-6.1$ & $R, I, z^{\prime}, J, K_{s}, 3.6,4.5$ & $17,9, \underline{6}, \mathbf{1 2}, 13, \mathbf{2 0}, 21,22$ \\
\hline $\mathrm{J} 1047+2124^{\mathrm{g}}$ & T6.5 & $880 \pm 76$ & -5.30 & $42 \pm 26$ & 1.77 & $12.1,(\mathbf{1 3 . 2})$ & $\sim 5.5$ & $\cdots$ & $\overline{4,9}, \mathbf{1 5}, 22, \mathbf{2 5}$ \\
\hline
\end{tabular}

Notes.

a The periods are quoted from the radio data, unless otherwise noted, and are consistent with the periods detected in photometric monitoring.

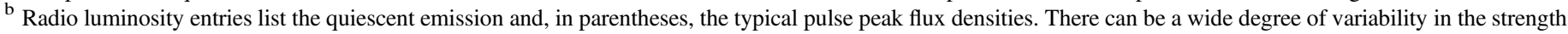

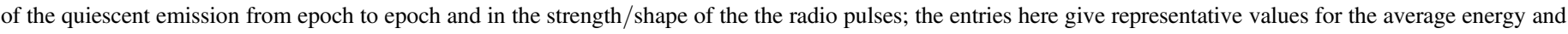
peak luminosity of the quiescent emission and pulses, respectively.

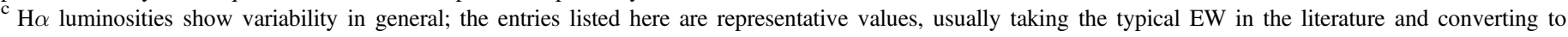
$L_{\mathrm{H} \alpha} / L_{\text {bol }}$ using the $\chi$ values of Schmidt et al. (2014b).

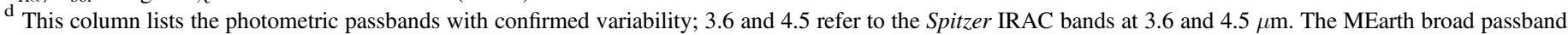
is in the red optical (Nutzman \& Charbonneau 2008).

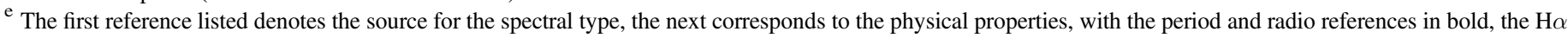
reference in italics, and the photometric variability reference underlined.

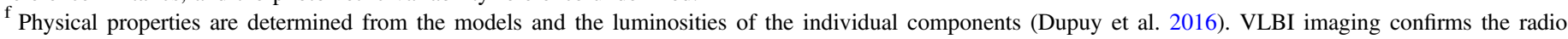

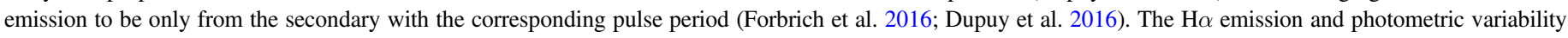
cannot be attributed to particular components.

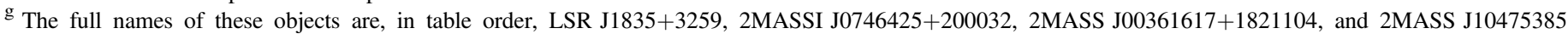
+2124234 .

${ }^{\mathrm{h}}$ Period precision is quoted by Wolszczan \& Route (2014) as $7 \mathrm{~ms}$.

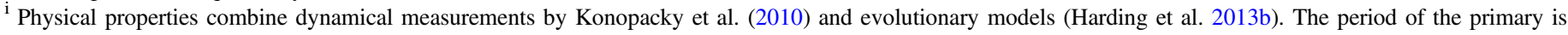

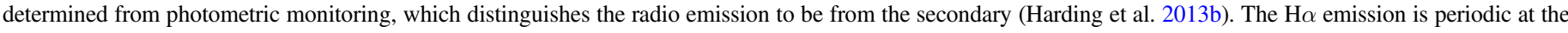
same rotation period as the radio emission (Berger et al. 2009).

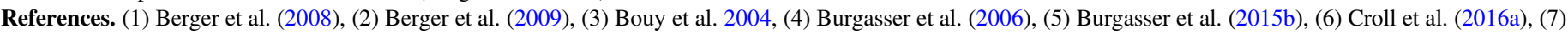

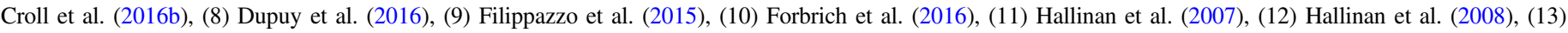

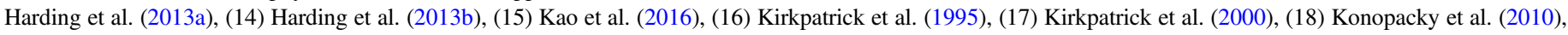

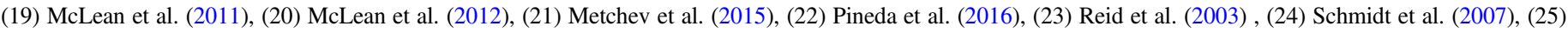
Williams \& Berger (2015), (26) Williams et al. (2015a), (27) Wolszczan \& Route (2014).

variability caused by magnetic spots and/or aurorae, and heterogeneous cloud cover; however, the relative importance of each across the UCD regime remains unclear. The possibility of long-lived storm systems in the cloudy atmospheres of brown dwarfs, adds an additional complication, potentially requiring concurrent multiwavelength data to disentangle, such as $\mathrm{H} \alpha$ emission in addition to photometric monitoring (Gizis et al. 2015). Although it is likely that clouds play a more important role for the coolest objects and that starspots may only be significant in the warmest UCDs, the nature of the transition from one to the other is an open question. Furthermore, as we will discuss in Section 3.3.2, photometric variability as a consequence of aurorae may be present throughout the UCD regime.

\section{Brown Dwarf Aurorae}

Brown dwarf aurorae provide a natural explanation for many of the observational trends in UCD magnetism. The characteristics of the periodic radio pulses-that they are highly circularly polarized with large brightness temperaturesindicate that the ECMI must be the emission mechanism (see Treumann 2006), and that the aurorae, the consequence of energy dissipation from stable large-scale magnetospheric current systems, are present throughout the UCD regime. However, the physical conditions that power the aurorae remain an open question. Here, we discuss the implications of potential auroral scenarios on multiwavelength brown dwarf emissions, how they fit within the observations of brown dwarf activity, and predictions based on the analogy with auroral systems in the gas-giant planets of the solar system. As a reference, we also compiled the physical properties and relevant observations of all of the potentially auroral UCD systems, based on their radio emission. This summary is presented in Tables 1 and 2 .

We discussed the dominant auroral electrodynamic engines in Section 1.2. In the case of brown dwarfs, the three engines, stellar wind, and satellite-induced and co-rotation breakdown, take on slightly different forms. Although we expect brown dwarfs in binary systems orbiting much larger stars to interact with the companion's stellar wind in much the same way that planets do around the Sun, for these brown dwarfs in large orbital separations $(\sim 10 \mathrm{~s}$ of $\mathrm{au})$, the production of significant aurorae is disfavored (Zarka 2007), while objects that may be sufficiently close are difficult to observe due to their small angular separation on the sky, requiring adaptive optics and/or very long baseline interferometry. Nevertheless, observationally many of the significant auroral brown dwarfs are isolated 
Table 2

Ultracool Dwarfs with Quiescent Radio Emission or Unconfirmed Radio Pulse Periodicity

\begin{tabular}{|c|c|c|c|c|c|c|c|c|}
\hline Object & SpT & $\begin{array}{l}T_{\text {eff }} \\
(\mathrm{K})\end{array}$ & $\begin{array}{c}{\left[L_{\mathrm{bol}}\right]} \\
\left(L_{\odot}\right)\end{array}$ & $\begin{array}{l}\text { Mass } \\
\left(M_{\text {Jup }}\right)\end{array}$ & $\left(\operatorname{erg~s}^{-1}{ }^{\left[L_{\nu}\right]} \mathrm{Hz}^{-1}\right)^{\mathrm{a}}$ & {$\left[L_{\mathrm{H} \alpha} / L_{\mathrm{bol}}\right]^{\mathrm{b}}$} & Phot. Var. ${ }^{c}$ & References $^{\mathrm{d}}$ \\
\hline J0952-1924 AB ${ }^{\text {e f }}$ & M7 & $\ldots$ & $\ldots$ & $\ldots$ & 14.4 & $\sim-4.0$ & $\ldots$ & $21, \mathbf{3 5}, 32$ \\
\hline LHS $3003^{\mathrm{g}}$ & M7 & $2595 \pm 29$ & -3.2 & $\ldots$ & 13.1 & $\sim-3.9$ & $\ldots$ & $4,18, \mathbf{5}, 41$ \\
\hline \multirow[t]{2}{*}{ LHS $2397 \mathrm{a}^{\mathrm{g}}$} & M8 & $2461 \pm 29$ & -3.3 & $\ldots$ & 13.2 & $\sim-4.0$ & $\ldots$ & $20,18,39, \mathbf{4 3}$ \\
\hline & L7.5 & $1330 \pm 29$ & -4.5 & $\ldots$ & $\ldots$ & $\ldots$ & $\ldots$ & \\
\hline $\mathrm{J} 1048-3956^{\mathrm{e}}$ & M8 & $2307 \pm 51$ & -3.51 & $77.00 \pm 10.37$ & $12.6,(\mathbf{1 4 . 8})$ & $\sim-4.7$ & $\ldots$ & $22,18, \mathbf{5}, \mathbf{3 4}, 10$ \\
\hline \multirow[t]{2}{*}{ LP $349-25^{\mathrm{h}}$} & M8 & $2660 \pm 30$ & -3.04 & $67 \pm 4$ & 13.9 & $\sim-4.6$ & $I, R$ & $19,16,27, \mathbf{3 5}$ \\
\hline & M9 & $2520 \pm 30$ & -3.18 & $59 \pm 4$ & $\ldots$ & $\ldots$ & $\ldots$ & \\
\hline LP 944-20 & M9 & $1942 \pm 144$ & -3.56 & $29.53 \pm 16.87$ & 12.6, (13.9) & $\sim-5.6$ & $\ldots$ & $14,18,10, \mathbf{3 4}, \mathbf{3 5}$ \\
\hline $\mathrm{J} 0024-0158^{\mathrm{e}}$ & M9.5 & $2390 \pm 80$ & -3.44 & $79.27 \pm 11.13$ & $12.8,(\mathbf{1 3 . 8})$ & $\sim-6.6$ & $<I$ & $33,18,2,10,27$ \\
\hline \multirow[t]{2}{*}{$\mathrm{J} 0720-0846^{\mathrm{e} g}$} & M9.5 & $2321 \pm 113$ & -3.51 & $85 \pm_{17}^{29}$ & $11.8,(\mathbf{1 2 . 9})$ & $\sim-4.8$ & $I+z$ & $11,9,18,9^{-}$ \\
\hline & $\mathrm{T} 5.5$ & $991 \pm 113$ & -4.82 & $65 \pm_{12}^{9}$ & $\ldots$ & $\ldots$ & $\ldots$ & \\
\hline $\mathrm{J} 1906+4011^{\text {e g i }}$ & L1 & $2102 \pm 113$ & -3.70 & $\ldots$ & 12.9 & $\sim-5.0$ & $K e p, i, z, 3.6,4.5$ & $23,18,24,25$ \\
\hline $\mathrm{J} 0523-1403^{\mathrm{e}}$ & $\mathrm{L} 2.5$ & $1939 \pm 68$ & -3.86 & $67 \pm 13$ & 13.6 & $\sim-6.2$ & $<I_{C}$ & $14,18, \mathbf{3}, 31, \overline{4} 2$ \\
\hline \multirow[t]{2}{*}{ GJ $1001 \mathrm{~B}^{\mathrm{g}}$} & L5 & $1581 \pm 113$ & -4.22 & $\ldots$ & 13.3 & $\sim-5.7$ & $\ldots$ & $38,18, \mathbf{3 4}, 42$ \\
\hline & L5 & $1581 \pm 113$ & -4.22 & $\ldots$ & $\ldots$ & $\ldots$ & $\ldots$ & \\
\hline \multirow[t]{2}{*}{$\mathrm{J} 1315-2649^{\mathrm{e} g}$} & L5 & $1581 \pm 113$ & -4.22 & $\cdots$ & 14.2 & $\sim-4.1$ & $<J,<K^{\prime}$ & $8,18, \mathbf{8}, 29,42$ \\
\hline & $\mathrm{T} 7$ & $825 \pm 113$ & -5.1 & $\ldots$ & $\ldots$ & $\ldots$ & $\ldots$ & \\
\hline \multirow[t]{2}{*}{$\mathrm{J} 0423-0414^{\mathrm{e} g \mathrm{j}}$} & L6 & $1483 \pm 113$ & -4.39 & $\ldots$ & $12.8,(\mathbf{1 3 . 7})$ & $\sim-5.9$ & $<I_{C}, J, K$ & $6,18,13,17,28,36,44$ \\
\hline & $\mathrm{T} 2$ & $1184 \pm 113$ & -4.62 & $\ldots$ & $\ldots$ & $\ldots$ & $\ldots$ & \\
\hline $\mathrm{J} 1043+2225^{\mathrm{e} g \mathrm{j}}$ & L8 & $1336 \pm 113$ & -4.50 & $\ldots$ & $12.7,(\mathbf{1 3 . 4})$ & $\sim-5.8$ & $\ldots$ & $15,18, \mathbf{2 8}, 36$ \\
\hline $\mathrm{J} 0607+2429^{\mathrm{e}} \mathrm{g}$ & L8 & $1336 \pm 113$ & -4.50 & $\ldots$ & 12.0 & $<-6.5$ & $<3.6,<4.5,<$ Kep & $12,18,26$ \\
\hline SIMP $0136+0933^{\text {e j k }}$ & $\mathrm{T} 2.5$ & $1089 \pm_{54}^{62}$ & -4.63 & $23 \pm_{13}^{16}$ & $12.2,(\mathbf{1 3 . 0})$ & $<-6.6$ & $J, H, K$ & $1,28, \mathbf{2 8}, 36,37$ \\
\hline $\mathrm{J} 1122+2550^{\text {e g j } 1}$ & T6 & $943 \pm 113$ & -4.9 & $\ldots$ & (14.9) & $\ldots$ & $\ldots$ & $30,18, \mathbf{4 0}-$ \\
\hline $\mathrm{J} 1237+6526^{\mathrm{e}}$ & T6.5 & $851 \pm 74$ & -5.36 & $40.85 \pm 25.96$ & $12.7,(\mathbf{1 3 . 1})$ & $\sim-4.2$ & $\ldots$ & $7,18,28,36$ \\
\hline
\end{tabular}

Notes.

${ }^{a}$ Radio luminosity entries list the quiescent emission and, in parentheses, the typical pulse peak flux densities. There can be a wide degree of variability; many of these objects have been observed multiple times, sometimes yielding non-detections. The entries here give representative values for the average energy and peak luminosity of the quiescent emission and pulses, respectively.

${ }^{\mathrm{b}} \mathrm{H} \alpha$ luminosities show variability in general; the entries listed here are representative values, usually taking the typical EW in the literature and converting to $L_{\mathrm{H} \alpha} / L_{\mathrm{bol}}$ using the $\chi$ values of Schmidt et al. (2014b).

${ }^{\mathrm{c}}$ This column lists the photometric passbands with confirmed variability; 3.6 and 4.5 refer to the Spitzer IRAC bands at 3.6 and $4.5 \mu \mathrm{m}$. "Kep" refers to the broadband optical passband of the Kepler mission. Entries prefaced by " $<$ " indicate constant fluxes during photometric monitoring observations.

d The first reference listed denotes the source for the spectral type, the next corresponds to the physical properties, with the radio references in bold, the H $\alpha$ reference in italics, and the photometric variability references underlined.

e The full names of these objects are, in table order, 2MASSW J0952219-192431, DENIS J1048.0-3956, 2MASSI J0024246-015819, 2MASS J07200325-0846499, 2MASS J19064801+4011089，２MASSI J0523382-140302，２MASS J13153094-2649513，２MASS J04234858-0414035， 2MASS J10430758+2225236, WISEP J060738.65+242953.4, SIMP J013656.5+093347.3, WISEP J112254.73+255021.5, and 2MASS J12373919+6526148.

${ }^{\mathrm{f}}$ This object is an unresolved spectroscopic binary-all measurements are from combined light (Reid et al. 2002; Siegler et al. 2005).

${ }^{\mathrm{g}}$ Entries for effective temperature and bolometric luminosity use polynomial relations from Filippazzo et al. (2015).

${ }^{\mathrm{h}}$ Although no period has yet been observed in the radio, a period from photometric variability yields $1.86 \pm 0.02 \mathrm{hr}$ (Harding et al. 2013a).

i Although no period has yet been observed in the radio, a period from photometric variability yields $8.9 \mathrm{hr}$ (Gizis et al. 2016).

j Objects have been observed to display multiple highly circularly polarized pulses, but a periodicity has not yet been determined.

${ }^{\mathrm{k}}$ Although no period has yet been observed in the radio, a period from photometric variability yields $\sim 2.4 \mathrm{hr}$ (Artigau et al. 2009).

${ }^{1}$ Follow-up observations since its initial radio detection have not revealed emission at the tentative period from the discovery paper by Williams et al. (2017).

References. (1) Artigau et al. (2006), (2) Berger (2002), (3) Berger (2006), (4) Bessell (1991), (5) Burgasser \& Putman (2005), (6) Burgasser et al. (2005), (7) Burgasser et al. (2006), (8) Burgasser et al. (2013), (9) Burgasser et al. (2015c), (10) Burgasser et al. (2015b), (11) Burgasser et al. (2015a), (12) Castro et al. (2013), (13) Clarke et al. (2008), (14) Cruz et al. (2003), (15) Cruz et al. (2007), (16) Dupuy et al. (2010), (17) Enoch et al. (2003), (18) Filippazzo et al. (2015), (19) Forveille et al. (2005), (20) Freed et al. (2003), (21) Gizis et al. (2000), (22) Gizis (2002), (23) Gizis et al. (2011), (24) Gizis et al. (2013), (25) Gizis et al. (2015), (26) Gizis et al. (2016), (27) Harding et al. (2013a), (28) Kao et al. (2016), (29) Khandrika et al. (2013), (30) Kirkpatrick et al. (2011), (31) Koen (2013), (32) Lee et al. (2010), (33) Leggett et al. (2001), (34) Lynch et al. (2016), (35) McLean et al. (2012), (36) Pineda et al. (2016), (37) Radigan et al. (2012), (38) Reid et al. (2008), (39) Reiners \& Basri (2010), (40) Route \& Wolszczan (2016a), (41) Schmidt et al. (2007), (42) Schmidt et al. (2015), (43) Williams et al. (2014), (44) Wilson et al. (2014).

systems in the field. Works by Schrijver (2009) and Nichols et al. (2012) suggest that motion through the ISM and reconnection in the large magnetosphere would not be able to produce enough energy to power the auroral radio emission. Both of these sets of authors suggest that co-rotation breakdown can provide sufficient energy to power the aurorae, with Hallinan et al. (2015) further suggesting that a satellite interaction could also provide sufficient energy. We discuss these two scenarios in Sections 3.1 and 3.2, respectively.
Regardless, an important ingredient in the emergence of auroral phenomena in brown dwarf systems is the presence of large-scale magnetic field topologies in some objects (see Section 2.1). The extended dipolar fields provide the conditions that generate an auroral electrodynamic engine, with the stronger fields at large distances enabling the strong coupling between the ionosphere and the middle magnetosphere. In contrast to the suggestions put forward in Williams et al. (2014), given the connection between aurorae and large-scale 
fields, the radio load (ECM) and X-ray-quiet objects likely host large-scale fields, while radio-quiet and X-ray flaring objects likely display strong small-scale fields (see Section 2.2.2). However, although a necessary component, the magnetic field topology may not be the distinguishing feature of the auroral processes (see Section 3.2).

\subsection{Co-rotation Breakdown}

For co-rotation breakdown, the magnetospheric current and energy dissipated is dependent on the differential velocity between the co-rotating magnetosphere and an extended plasma disk, the electron density of that disk, and the strength of the brown dwarf's magnetic field (Cowley \& Bunce 2001; Nichols et al. 2012). Furthermore, these properties also set the location of the auroral oval with respect to the magnetic axis and hence the particular flux tubes connected to the shearing layer (Cowley \& Bunce 2001). Thus, the magnetic field strength and rotational velocity play a crucial role in the generation mechanism and energy dissipation in this scenario. This electrodynamic engine would predict a strong dependence on rotational velocities and magnetic field strengths, both of which are much larger in brown dwarfs compared to Jupiter and could lead to much stronger aurorae in brown dwarfs (Hallinan et al. 2015). As seen in Figure 8, the radio detection fraction of UCDs rises in the more rapidly rotating objects, consistent with rapid rotation, $P \lesssim 3-4 \mathrm{hr}$ (see Figure 8 ), as a potentially critical ingredient to the generation of strong radio emission. The strength of stable auroral current systems also depends on the conductivity of the brown dwarf ionosphere (Cowley \& Bunce 2001; Nichols et al. 2012). Although there are many variables involved, including the self-ionization from the electron beam, this would indicate a dependence on $T_{\text {eff }}$ for the strength of auroral emissions. There is some hint of this in the quiescent radio emission of the auroral targets in Table 1, but larger samples are needed. Additionally, co-rotation breakdown predicts that auroral atmospheric emission should be predominantly confined to a narrow oval around the magnetic axis; we discuss these atmospheric effects and their observability in Section 3.3.

However, a significant unknown for brown dwarfs is the existence of an extended equatorial plasma sheet that seeds the magnetospheric currents with plasma. Many possibilities for loading the magnetosphere with plasma have been put forward, including interaction with the ISM, reconnection events at the photosphere early in the brown dwarf's evolution, and ablation of the atmosphere due to auroral currents (Hallinan et al. 2015). As a comparison, in the Jovian system, Io's volcanic activity provides the material to load the plasma disk. Analogous systems have not yet been confirmed around very low-mass stars and brown dwarfs; however, there have been recent detections of small potentially rocky planets around UCDs with significant tidal interactions (Udalski et al. 2015; Gillon et al. 2016, 2017). Moreover, models of planet formation around very low-mass stars suggest that these planets can form frequently and are likely to have radii similar to that of Earth (Alibert \& Benz 2017; He et al. 2017).

\subsection{The Possible Role of Planetary Companions}

Given these new planetary detections, the satellite-induced scenario for the electrodynamic engine becomes an intriguing possibility. The satellite flux tube interaction is generated by the differential motion of an orbiting satellite through the brown dwarf's magnetosphere. In this scenario, the auroral power is a function of this velocity, the magnetic field strength at the orbital location of the satellite, and the cross-sectional area of interaction of the planet, usually defined by the size of the obstacle (Zarka 2007). For a rocky planet without an intrinsic magnetic field, the cross-section is determined by the planet's exo-ionosphere (e.g., Io), whereas for a system with its own magnetosphere, the area is determined by the magnetopause distance of the planet within the brown dwarf's magnetosphere (e.g., Ganymede, Zarka 2007). Thus, stronger emission is expected for closer-in planetary systems, faster rotators, stronger magnetic field brown dwarfs, and larger, more magnetized planets. Although many of these physical properties are unknown, using typical values, Hallinan et al. (2015) note that a planet orbiting in a $\sim 1.25$ day orbit could generate enough energy to power auroral UCD emissions. Interestingly, under this scenario, the auroral emission may provide a separate constraint on the satellite radius and provide a way to potentially confirm the existence and strength of the planetary magnetic field. Regardless, measurements of the surface feature and the emissions (see Section 3.3) associated with the flux tube can provide a way to distinguish the satellite scenario from co-rotation breakdown.

Nevertheless, because the origin of the magnetospheric plasma is an open question, the presence of planetary companions to brown dwarfs could be an important ingredient in both the satellite and co-rotation breakdown scenarios, which may additionally overlap in any given system. If the magnetospheric plasma is generated by some other means, then co-rotation breakdown may be the dominant brown dwarf auroral engine. While the discovery of a planet directly tied to auroral emissions in a brown dwarf would provide strong evidence for the importance of the satellites, an examination of the statistics of auroral objects and the occurrence rate of closein planets around them may provide important clues regarding the underlying auroral mechanisms. If the occurrence rate of planets around auroral UCDs is significantly larger than the rate for the whole population of rapidly rotating UCDs, these data would be suggestive of the important role planetary companions play in auroral UCD systems. Recently, He et al. (2017) showed that the occurrence rate of rocky planets around brown dwarfs with orbital periods less than 1.28 days is $\lesssim 67 \%$, and based on recent planetary transit detections, may be $\sim 27 \%$. This is at least consistent with a lower limit of $\sim 10 \%$ from auroral radio and $\mathrm{H} \alpha$ emission, if all of these brown dwarfs hosted unconfirmed planetary systems. When making this comparison, however, it may be important to also take into account the planetary system architectures. While a single very close-in planet may suffice to either seed the plasma disk or provide the obstacle for the auroral flux tube, tidal interactions may spin down the brown dwarf host, possibly limiting its ability to power significant aurorae, although this would depend on substantial unknowns, such as the degree of brown dwarf tidal dissipation (Ribas et al. 2016; Gillon et al. 2017). In contrast, interactions in multiple-satellite systems, such as the one around Jupiter, can prevent the total tidal synchronization of the close-in satellites through resonant orbital interactions and a pumping of the orbital eccentricity (see Peale 1999 and references therein). These effects could sustain significant tidal interactions throughout the system age that contribute to the internal satellite heat flux and the generation of prominent 
volcanism, and consequently a plasma disk, without significantly spinning down the host brown dwarf; for example, the Jovian rotational period is $\sim 10 \mathrm{hr}$ (Bagenal et al. 2014). If planetary companions are a necessary condition, these additional considerations may drive the difference between the overall UCD planet occurrence rate and the prevalence of UCD auroral emissions. Current and future surveys, such as TRAPPIST and TESS, will provide a better understanding of planet statistics in the UCD regime with which to compare the aurorally active set of brown dwarfs (Gillon et al. 2011; Ricker et al. 2014).

An important example potentially illustrating the need for these distinct conditions to drive the electrodynamic engine, whether induced by satellite interactions and/or co-rotation breakdown, is the binary NLTT 33370 . This object, composed of two nearly identical M7 dwarfs (see Table 1) at a separation of 2.528 au (146.6 mas on the sky; Dupuy et al. 2016), has been studied extensively across different wavelengths to characterize its magnetic emissions (Williams et al. 2015a). This object shows the highly circularly polarized radio pulses characteristic of the ECMI; however, radio emission is observed in only one of the two components, the secondary, as shown in VLBI imaging (Forbrich et al. 2016). The two binary components also appear to have similar rotational velocities $(v \sin i \sim 45$ $\mathrm{km} \mathrm{s}^{-1}$ ), consistent with the radio period and the photometric period measurements (McLean et al. 2011; Williams et al. 2015a; Forbrich et al. 2016). That these two nearly identical objects, with the same mass, age, luminosity, effective temperature, and rotation period, display such different radio emission properties is difficult to explain without a significant underlying difference in the fundamental conditions that generate the radio emission. These observations can be explained by an auroral electrodynamic engine operating around NLTT 33370 B and not NLTT 33370 A. Forbrich et al. (2016) further use their astrometric monitoring to rule out Jupiter-mass companions in the NLTT 33370 AB system in a wide variety of orbits; however, a smaller rocky planet may be sufficient to provide the conditions to drive an auroral engine. Similarly, the L0.5+L1.5 binary 2MASSI J0746425+200032 with separation 2.9 au (237.3 mas on the sky; Konopacky et al. 2010) shows periodic radio and $\mathrm{H} \alpha$ emission at the same $2.072 \mathrm{hr}$ period, attributable to the secondary, while the primary has a confirmed photometric period of $3.32 \mathrm{hr}$ (Berger et al. 2009; Harding et al. 2013b). Like NLTT 33370, the components of 2MASS J0746+2000 have similar physical properties but very different magnetic emissions. Planet searches around these objects would be potentially very interesting. An Earthmass planet in a circular 1 day orbit around these objects, for example, would have a radial velocity semi-amplitude up to $\sim 3.5 \mathrm{~m} \mathrm{~s}^{-1}$. RV signals like these will be searched for in late M-dwarf targets by upcoming NIR high-resolution spectrographs, such as the Habitable-Zone Planet Finder (Mahadevan et al. 2012). Further study of these and other benchmark objects will be key in disentangling the nature of the auroral processes.

\subsection{Impact on Atmosphere}

If ECMI is present, then a strong-field-aligned current is driving an electron beam to precipitate into the atmosphere. This has a significant impact on the brown dwarf atmosphere, leading to the creation of multiwavelength auroral emissions. The same physical processes that take place in the Jovian system should operate in brown dwarfs (see Section 1.2), although important differences may arise due to the different atmospheric conditions and potentially due to the different properties of the auroral electron beams.

\subsubsection{Emission Line Features}

The collision of high-energy electrons with an atmosphere predominantly of neutral $\mathrm{H} / \mathrm{H}_{2}$, as is found in brown dwarfs, leads to the ionization and excitation of the hydrogen gas. The de-excitation and recombination of hydrogen will lead to considerable Balmer and Lyman series emission, as well as UV emission in the Lyman and Werner bands (Badman et al. 2015). Consequently, brown dwarfs hosting auroral radio emission are likely to generate surface features that are bright in $\mathrm{H} \alpha$, Ly $\alpha$, and the FUV. Thus, the presence of these emissions should be correlated with the presence of ECM radio emission. The statistics of radio detections and $\mathrm{H} \alpha$ emission in late L-dwarfs and T-dwarfs show evidence for this correlation (Kao et al. 2016; Pineda et al. 2016). The auroral FUV emission in brown dwarfs has not yet been discovered, but would be an important confirmation of the effects of auroral electron beams in brown dwarf atmospheres. Moreover, following the Jovian example, this FUV emission would be diagnostic of the total auroral energy and is sensitive to the auroral electron energy distribution (Bhardwaj \& Gladstone 2000; Badman et al. 2015; J. S. Pineda et al. 2017, in preparation).

Additionally, the nature of the excitation mechanism for the hydrogen emission features could potentially be used as a diagnostic of the auroral process. Detailed modeling is required to predict the Balmer line spectrum from the electron impacts on the brown dwarf; however, the emission ratios of the lines likely deviate from expectations from case B recombination and simple LTE gas models. An analysis of the Balmer series emission line ratios (decrement) by Stelzer et al. (2012) for one of the late M-dwarfs with strong radio emission showed that these models proved to be a poor fit to the data and did not follow the expectations from a standard stellar chromospheric perspective. An auroral NLTE origin for the Balmer series emission could account for this discrepancy. More detailed observations of the emission lines of auroral brown dwarfs would aid in this endeavor.

Depending on the auroral electrodynamic engine, these features will appear as ovals around the magnetic axis or be localized to the satellite flux tube footpoint, which have different observational signatures. The different morphologies of the surface features will be imprinted on the emission lines, like $\mathrm{H} \alpha$. In contrast to chromospheric emission, which will be fully rotationally broadened, the line profile of the auroral $\mathrm{H} \alpha$ emission will be determined by the size and location of the auroral surface feature. Emission localized at a flux tube footpoint would be narrow and centered at velocities set by the longitude of the surface feature. The emission features of an auroral oval would be wider and span a range of velocities consistent with its $\mathrm{L}_{\text {-shell }}{ }^{4}$ and the tilt of the magnetic axis relative to the rotational axis. The shape of the line would also deviate from a simple velocity-broadened Gaussian profile because the emission at the center of the oval would be missing. High-resolution spectroscopic observations of auroral brown dwarf emission features can be used to reconstruct these

\footnotetext{
4 The L-shell is the distance, in units of the object's radius, along the magnetic equator that maps to a location on the stellar surface along the corresponding field line traversing the magnetic equator.
} 
properties of the surface features (J. S. Pineda et al. 2017, in preparation).

The observed variability also depends on the auroral engine. For an auroral oval, the feature rotates around as the magnetic axis rotates around. Depending on the viewing geometry, the auroral surface emission could rotate in and out of view, creating a sinusoidal signal, like what is observed on LSR $\mathrm{J} 1835+3259$ (Hallinan et al. 2015). This variability is also imprinted on the velocity profile of the emission lines. In the case of a flux tube footpoint, the time variability of the emission is additionally modulated by the orbital motion of the satellite in addition to the rotational period of the object. This leads to long-term variability of the emission features. Emission line variability in excess of the rotational period has already been confirmed in one auroral brown dwarf (Pineda et al. 2016). Like the Jovian system, the auroral emission features are likely to be intrinsically variable on short timescales, reflecting changes in the electron beam energy distribution and auroral current system. Stellar chromospheric emission is also characterized by intermittent variability and can show enhanced emission within starspot regions (Reiners \& Basri 2008; Lee et al. 2010).

As discussed in Section 1.2, the ionization of molecular hydrogen from electron impacts leads to the creation of ionized triatomic hydrogen. $\mathrm{H}_{3}^{+}$, however, is sensitive to the conditions in the atmosphere, and could be an important diagnostic with strong emission features at $2 \mu \mathrm{m}$ and $4 \mu \mathrm{m}$ (Tao et al. 2011, 2012; Badman et al. 2015). The ro-vibrational features are thermally excited and depend on the atmospheric temperature. Additionally, the presence of $\mathrm{H}_{3}^{+}$is limited by the electron number density and the concentration of gas species, like $\mathrm{CH}_{4}$ and $\mathrm{H}_{2} \mathrm{O}$, that act to destroy the ion (Badman et al. 2015). Interestingly, although auroral electron beams in brown dwarfs are likely to create $\mathrm{H}_{3}^{+}$, the concentration may not build up significantly if the ion is quickly destroyed. This would occur for large ionization fractions (higher in brown dwarfs relative to Jupiter but still mostly neutral; see Section 2.2) or a beam with high mean electron energies that penetrates to the deep atmospheric layers, below the homopause, where the concentration of molecules is high. Observations of these features could potentially be used to constrain the brown dwarf auroral beam electron energy distribution (J. S. Pineda et al. 2017 , in preparation). If the auroral brown dwarfs do host significant $\mathrm{H}_{3}^{+}$emissions, it may be possible to observe them with the upcoming James Webb Space Telescope. This could also provide an additional diagnostic that would distinguish between an aurorally active UCD and one hosting a chromosphere/corona.

Because these emission features depend on the atmospheric conditions, like the Jovian system, their intensity is a diagnostic of the upper atmospheres of brown dwarfs. If detected, features like the $\mathrm{H}_{2}$ Werner band emission and $\mathrm{H}_{3}^{+}$lines would provide the only probe of these atmospheric regions well above the photosphere; however, the effect will require detailed modeling to understand thoroughly.

\subsubsection{Photometric Variability}

The impact of the electron beam on the atmosphere deposits large amounts of energy, heating the atmosphere. In Jupiter, the bulk of the auroral energy emerges as thermal emission (see Section 1.2). The effect of this heating will depend on the atmospheric layers, where the bulk of the energy deposition takes place. Studies examining the effect of thermal perturbations in brown dwarf atmospheres have illustrated the wavelength dependence of the variable emission and demonstrated that stronger variability at IR wavelengths is generated when the energy is deposited higher in the atmosphere (Morley et al. 2014; Robinson \& Marley 2014). In addition to changing the temperature profile, the auroral energy alters the chemical structure and can impact the opacity of the auroral surface region. Hallinan et al. (2015) argues that this may be the mechanism generating the auroral surface features seen in broadband optical monitoring of auroral brown dwarfs (Harding et al. 2013a).

Modeling the auroral electron beam impact is key to understanding these effects. However, there are several expectations with regard to the photometric variability of auroral surface regions. The variability is generated near the magnetic axis and may be preferentially located near the rotational axis, generating sinusoidal variations as the feature moves in and out of view. The surface features are relatively steady and remain present on long timescales, exceeding the transient signals expected individually from starspots and/or variable cloud structures. These features can potentially be distinguished from long-lived storm systems (akin to Jupiter's great spot) through their latitudinal distributions; the storms are more prevalent along the equatorial belts, whereas the auroral variability is likely at high latitudes near the rotational axis. There should also be a strong correlation between objects displaying auroral radio emission and those showing long-term periodic variability. Comparing those objects with large NIR variability, in the $J$ and $K$ bands, to those with auroral radio emission and/or $\mathrm{H} \alpha$ emission shows a mixed record. The canonical L/T transition variables 2MASS J21392676 +0220226 and SIMP J013656.5+093347.3, for example, do not display $\mathrm{H} \alpha$ emission. This is somewhat surprising, since SIMP $0136+09$ was detected with highly circularly polarized radio emission; however, this may point toward the influence of a satellite (see Section 3.3.1; Kao et al. 2016; Pineda et al. 2016). Other objects like Luhman16 do show photometric variability and no sign of auroral activity (Osten et al. 2015). Some objects with IR variability from Spitzer, on the other hand, do show activity, like 2MASS J00361617+1821104 (Metchev et al. 2015). Long-term monitoring of these objects may be needed to distinguishing the mechanisms producing the surface features, but there does not appear to be a one-to-one connection between the auroral activity and photometric variability (see also Miles-Páez et al. 2017). The data are consistent with two independent effects taking place on similar populations of objects, with general variability more common than auroral activity, and their interplay being responsible for the observed phenomena in some objects throughout the UCD regime (Croll et al. 2016a).

\subsection{Auroral Beaming Geometry and Radio Emission}

The different proposed electrodynamic engines of auroral emission produce distinct geometric beaming patterns. To understand this better, we modeled the radio emission patterns for both a uniform auroral ring and a single auroral flux tube to approximate the ECMI radio sources generated by co-rotation breakdown and the magnetosphere-satellite interaction, respectively. We visualize these radio sources in Figure 11. The left panel of the plot shows the locations of the auroral radio rings, situated around the magnetic axis in both the northern and 

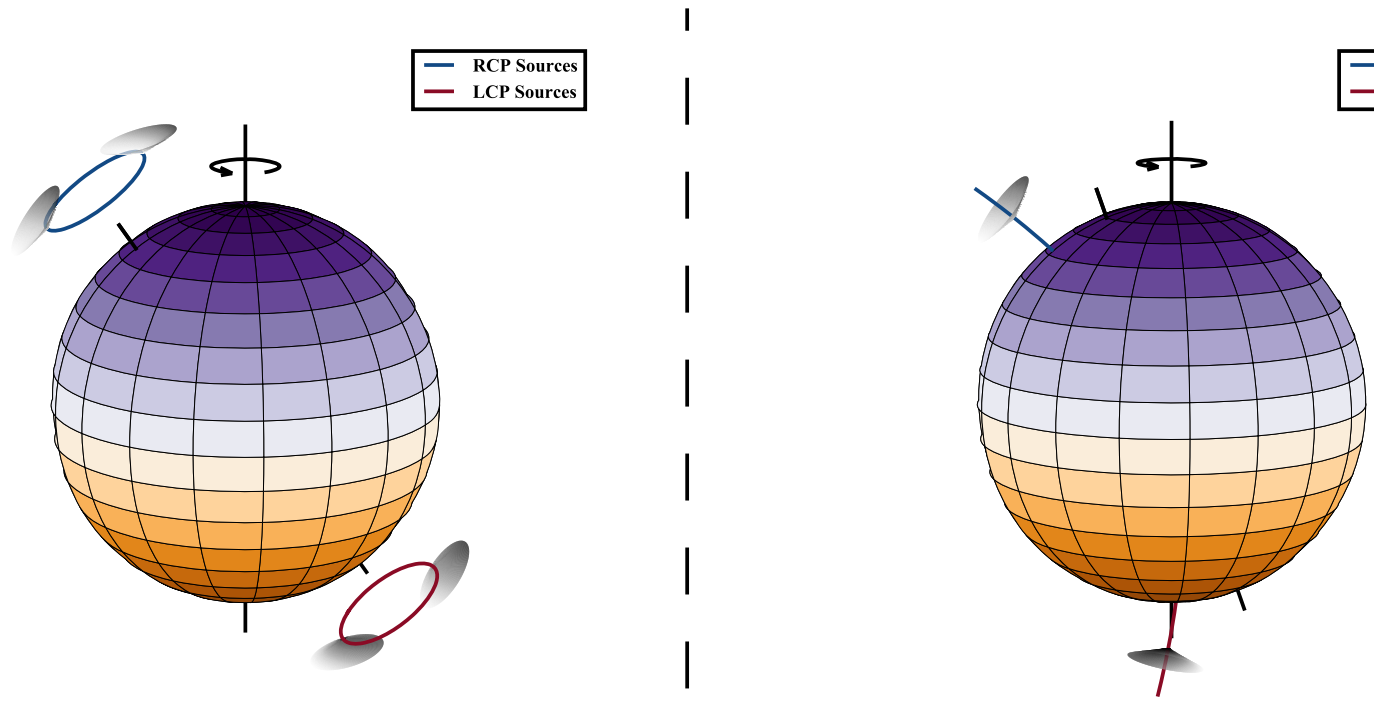

I

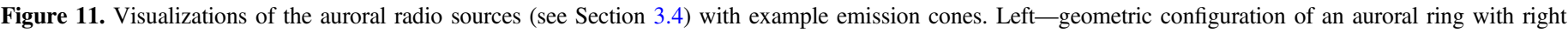

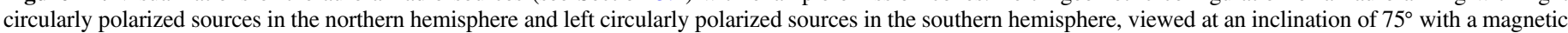

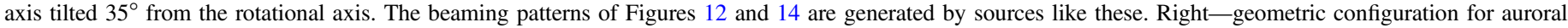

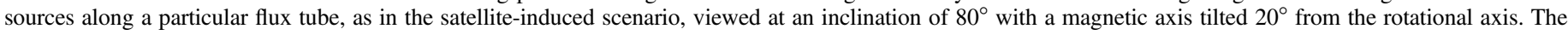
beaming patterns of Figure 15 are produced by these auroral sources.

southern hemispheres, while the right panel shows the emitting field lines for a satellite flux tube. Overlaid are illustrative hollow radio emission cones (see Section 1.2). At each point along the blue and red lines of the plots in Figure 11, we consider there to be an ECMI radio emission source and model the resulting cumulative beam pattern on the sky as seen from the brown dwarf.

Modeling the brown dwarf radio emission on what is observed from Jupiter, we can use some basic properties of the emission to determine what to expect in the beaming pattern and the variability from brown dwarfs. The Jovian radio aurorae are beamed into thin $\left(\sim 1^{\circ}-2^{\circ}\right)$ hollow cones, emitting in directions nearly perpendicular to the local magnetic field direction, with cone half-angles $\sim 80^{\circ}-90^{\circ}$ (Treumann 2006). The emission is highly circularly polarized and appears to show different directions of polarization from the different magnetic poles, consistent with X-mode waves in the plasma (Zarka 1998). We take the large-scale field to be predominantly dipolar and use it to map the acceleration regions to different locations in the magnetosphere. With these assumptions, we can examine the expected beam patterns for different systems and radiation sources, and what effect these generic properties have on the observations of auroral emission. By contrast, numerous authors have used these similar assumptions to try to directly constrain the properties of individual objects through models of their dynamic spectra (Yu et al. 2011; Lynch et al. 2015; Leto et al. 2016). Although interesting, these efforts require many assumptions of typically uncertain parameters for a problem that is difficult to answer accurately, even with much more information, as has been attempted with radio observations of Jupiter (see Hess et al. 2011).

In the case of co-rotation breakdown, the ECMI sources are expected to be concentrated in a ring, near the object's surface, around the magnetic axis, with the location defined by the L-shell associated with the magnetospheric current system. In Figure 12, we show a model beam pattern on the sky, in longitude and latitude, as viewed from the brown dwarf in a two- dimensional projection. The emission sources are from an auroral ring in the north and south hemispheres, similar to the left panel of Figure 11. The pattern shows the intensity as a function of position on the sky, using both right-hand circular polarization (positive intensities) and left-hand circular polarization (negative intensities). The inclination is set to $90^{\circ}$ with the magnetic axis tilted at an angle of $20^{\circ}$ in a uniform ring close to the surface $\left(1.4 R_{*}\right)$ with an L-shell of 30 , corresponding to sources located $\sim 12.5$ from the magnetic axis. The pattern of emission encompasses a large swath of the total sky. The peak emission intensities are generated at the edge of the beam pattern where the contributions from sources at multiple points along the ring contribute constructively. As the object rotates, this pattern traverses the sky in longitude and generates the periodic variability of the ECM emission.

For the observer, they would see very different light curves depending on their geometric orientation with respect to this beaming pattern. As plotted in Figure 12, the pattern uses an inclination of $90^{\circ}$, and the observer sees the light curve generated by the horizontal cut of this pattern at $0^{\circ}$ latitude. However, other inclination light curves can be read directly from these figures by looking at the latitudinal cut corresponding to $90^{\circ}-i$. We plot some example light curves in Figure 13 for a selection of viewing inclinations from Figure 12. If the object is viewed at an inclination of $0^{\circ}$, viewed above the rotational axis, there is no ECM emission visible. Consequently, the beaming pattern and the viewing geometry can have a significant impact on the detection statistics for radio surveys of brown dwarfs. At other inclinations, the variability encompasses multiple pulses, a single pulse, and/or multiple polarizations. Thus, a variety of light curve morphologies are possible and can change depending on the particular emission parameters, like cone width and cone opening angle. A narrower cone width, for example, would produce sharper pulse features. Comparing the morphology to the radio light curves produced in various surveys, we see a broad similarity between these shapes and the 


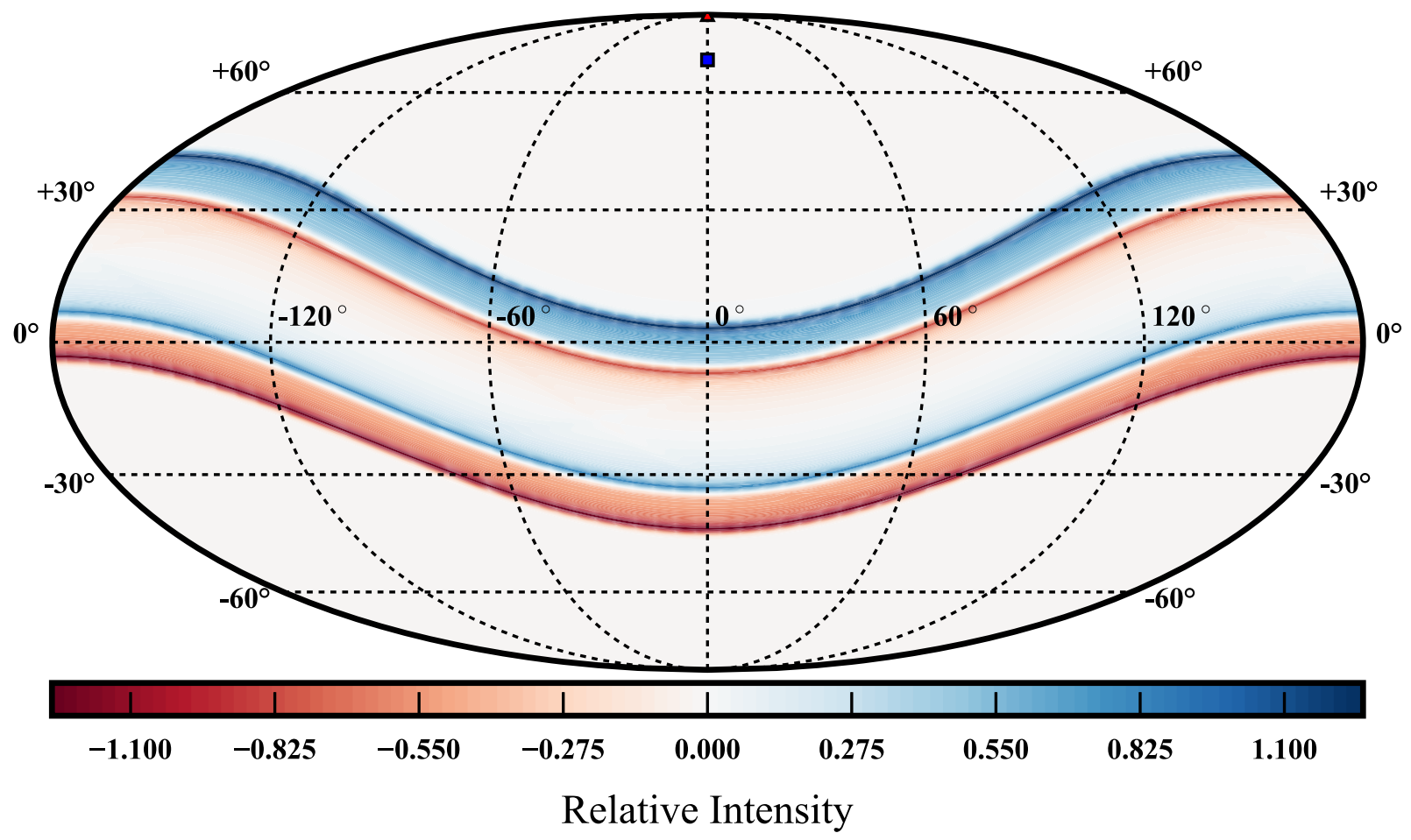

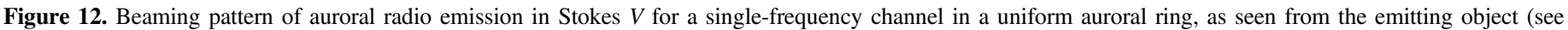

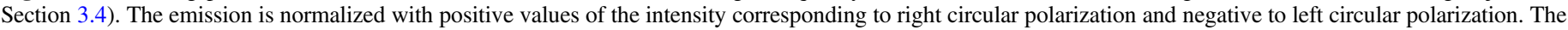

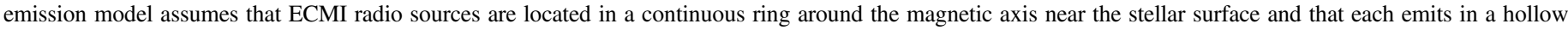

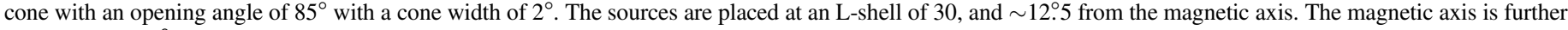

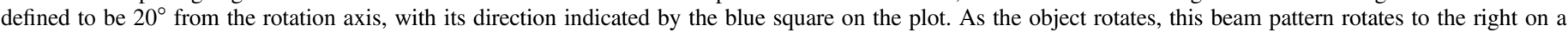
fixed sky.

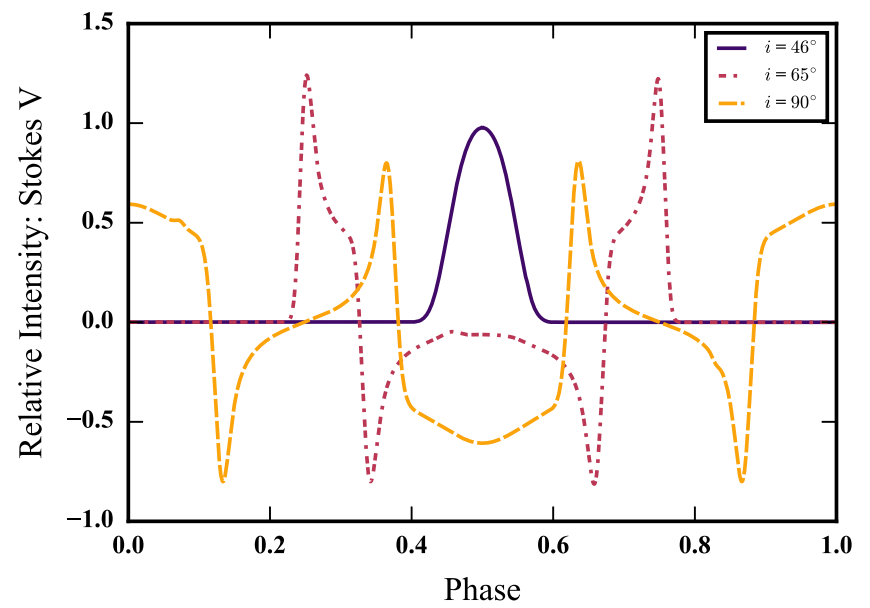

Figure 13. Intensity light curves in Stokes $\mathrm{V}$ from the variable ECM radio emission beam pattern of Figure 12, as viewed with different object inclinations. These light curves correspond to latitudinal cuts in the normalized intensity pattern created from the rotational variation of the emission. Phase 0 corresponds to longitude $0^{\circ}$ in Figure 12 . The beam pattern can generate a broad variety of light curves depending on the relative geometry of the object and the observer.

generic light curves of our model, based on basic assumptions about the emission process (Hallinan et al. 2008; Berger et al. 2009; Williams \& Berger 2015; Kao et al. 2016). The beam pattern in Figure 12 uses uniform rings of equal intensities, but observations of Jupiter indicate that the auroral emission can fluctuate (see Zarka 1998); moreover, the rings may not be populated uniformly with sources, leading to gaps in the light curves and variable pulse intensities and asymmetries between the right and left circularly polarized emissions. Thus, although an auroral ring may be predicted to produce multiple detectable pulses of both polarizations in a single period, they may not always be visible. However, if they are seen, then the separation of the pulses can be used to constrain the cone opening angle and the orientation of the magnetic axis relative to the rotational axis, keeping in mind the various degeneracies of doing that inversion.

An examination of these beaming patterns reveals that they encompass a large fraction of the sky as the object rotates around. Moreover, the fraction is larger when the magnetic axis is misaligned with respect to the rotational axis. In Figure 14, we show a beam pattern similar to that of Figure 12, but with a magnetic axis $35^{\circ}$ misaligned instead of $20^{\circ}$, where the pattern encompasses two-thirds of the sky. The proportion depends on the L-shell of the radio sources, the source height, and the cone opening angle, but is large for reasonable values of these parameters, $\sim 50 \%$. Pineda et al. (2016) and Kao et al. (2016) argue that the large overlap between objects with radio auroral emissions and optical auroral emissions indicates that the geometric selection effect biasing the radio detections might not be very strong. If this is the case, and the fraction of auroral objects is driven largely by the proportion of objects with physical conditions amenable to the generation of auroral magnetospheric currents, then generically, the magnetic axes of brown dwarfs are likely to be misaligned or at least a substantial component of the large-scale field is misaligned. Given these considerations, the detection of quiescent radio emission from a pole-on L-dwarf is unlikely to generate pulsed radio emission unless the magnetic axis is totally misaligned (Gizis et al. 2016). Similarly, the detection of variable circularly polarized emission in the data of WISEP J112254.73+255021.5 


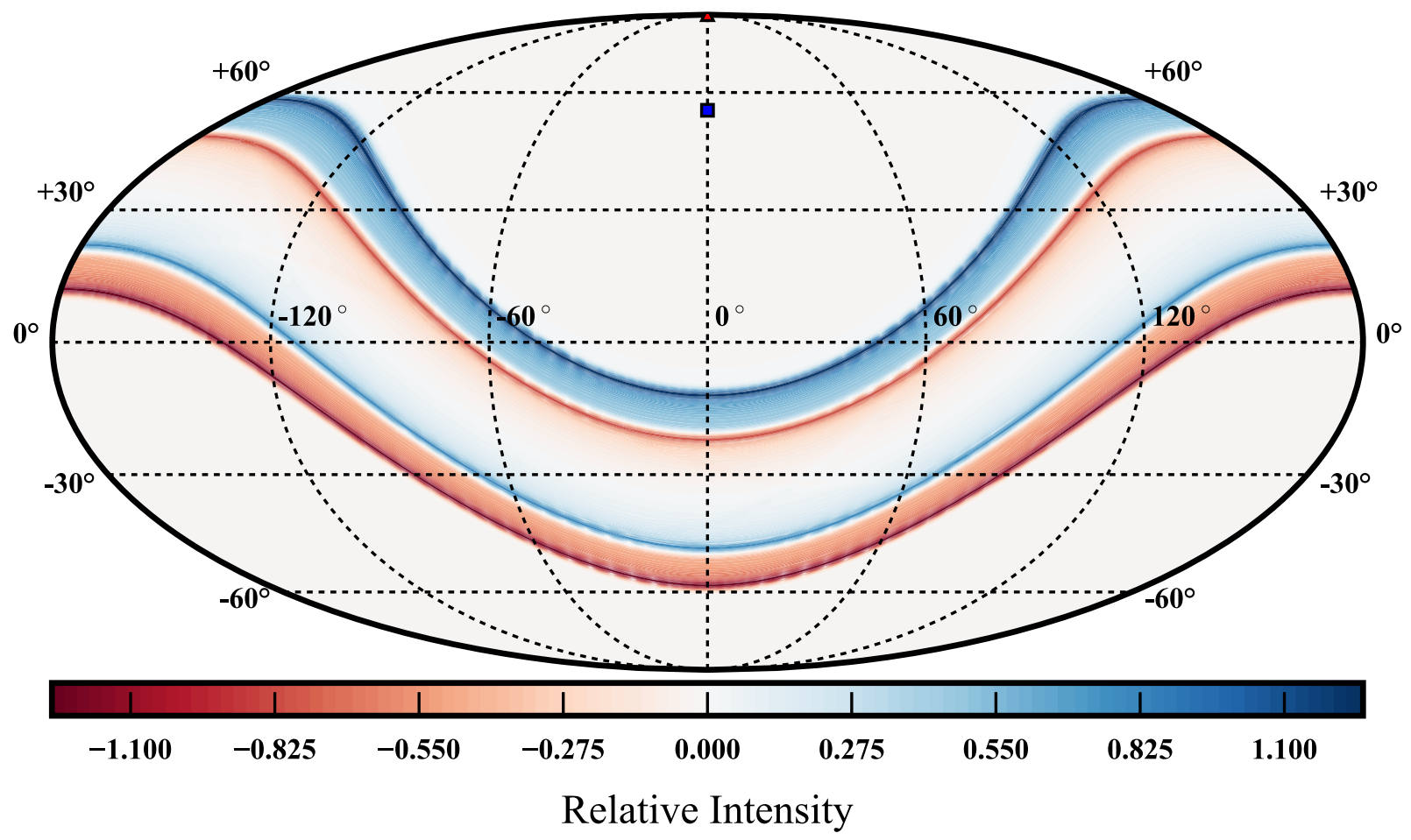

Figure 14. Same as Figure 12, but with the tilt of the magnetic axis set to $35^{\circ}$. With a larger angle between the rotational and magnetic axes, the proportion of the sky that is traversed by the auroral beam pattern increases.

has been used to argue for a misaligned magnetic axis in that T-dwarf (Williams et al. 2017). This is particularly interesting because our model light curves predict, in these cases, some circularly polarized emission between the peak pulses with relatively uniform auroral rings.

In contrast to the auroral oval case, the generation of ECMI through currents in a flux tube connecting a satellite and a brown dwarf produces a very different beam pattern and variability signal. Under these circumstances, the radio source region is confined to the longitude associated with the flux tube of the satellite (see the right panel of Figure 11). However, the properties of the individual radio sources should be the same: wide and thin hollow cones with different polarizations in the northern and southern hemispheres. In Figure 15, we show an example beam pattern for a satellite-induced source region with an L-shell of 10, spanning several heights analogous to multiple frequencies (i.e., $3-12 \mathrm{GHz}$ ) and two different instances of the rotational period, assuming the planet has remained relatively fixed at a longitude of $0^{\circ}$. In the top panel, the direction of the magnetic dipole is pointing toward the satellite, and in the bottom panel it is pointing away, a 0.5 difference in rotational phase. There are several effects illustrated in this diagram. The inclusion of several frequencies, in contrast to the single-frequency beam patterns of Figures 12 and 14, broadens the pulses when examined in broadband light curves. Since the different frequencies map to different field strengths, the corresponding emission cones are pointed in slightly different directions, creating a slight broadening of the broadband ECMI pulse and slightly different arrival times for the emission at different frequencies as the emission cones sweep into view during the rotational period of the brown dwarf. We do not consider the effect of the frequency-dependent
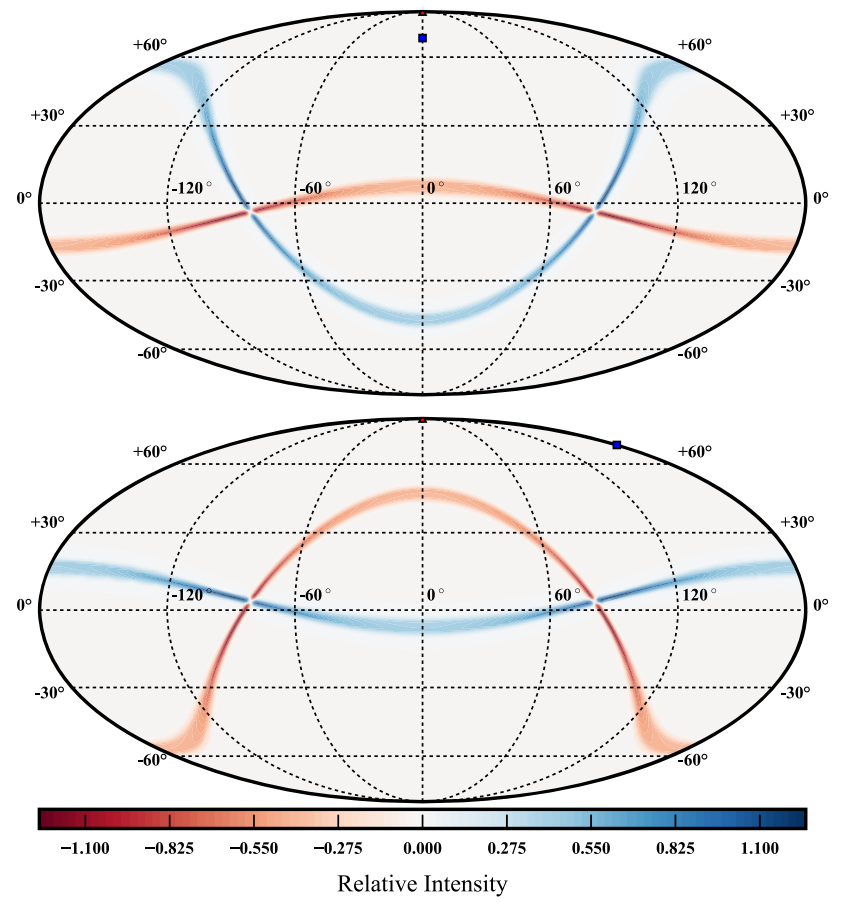

Figure 15. Emission beam patterns on the sky, as in Figure 12, for a set of ECMI radio sources at a fixed longitude associated with a current system fixed to a brown dwarf satellite flux tube. The range of sources span emission frequencies 3-12 GHz, for a dipolar field strength of $4.5 \mathrm{kG}$ at the brown dwarf surface along the magnetic axis. The L-shell is set to 10 and the magnetic axis is tilted by $20^{\circ}$. The top plot is oriented with the magnetic axis, indicated by the blue box, pointing toward the direction of the planet, and the bottom plot differs in phase by $180^{\circ}$, with the axis pointing away from the planet. The emission pattern transforms continuously on the sky with the rotation and orbital motion of the satellite. 


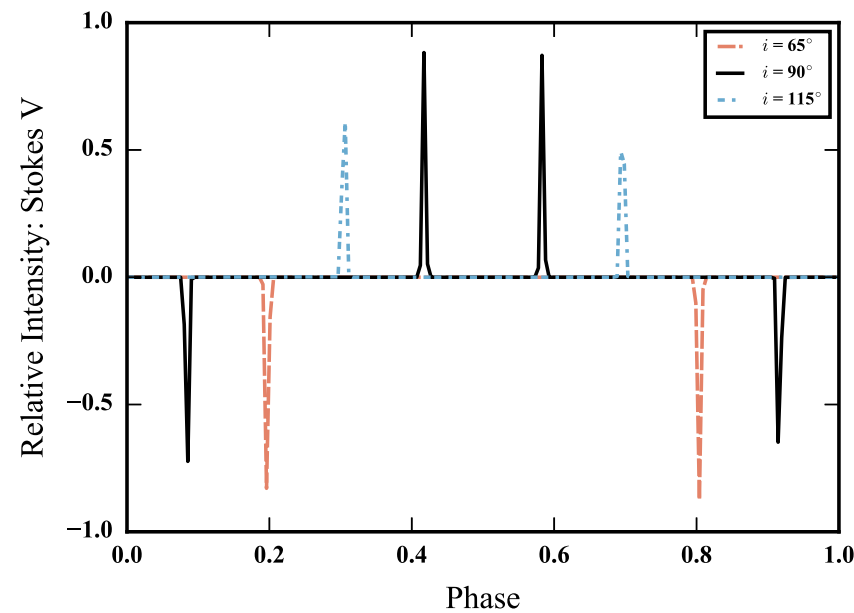

Figure 16. Radio light curves of single-frequency channels in Stokes $V$ intensity with the object's rotation for different observers viewing a satelliteinduced auroral radio source, as in Figure 15. The observers see narrow pulses when the beam pattern traverses their line of sight, and depending on the viewing geometry, the observer may see only one polarization or the other, or both polarizations coming from the north and south hemispheres, respectively. $i>90^{\circ}$ corresponds to the object's rotational axis pointing away from the observer.

refraction of the radiation, which generates more significant deflections at lower frequencies, as it propagates out of the ECM emission region (Mutel et al. 2008). Attempting to do so requires further assumptions about the plasma density in the magnetosphere, which has little observational constraints; however, including this physical effect would narrow the emission beam patterns (less instantaneous sky coverage) for the lower energy radiation, perhaps leading to observing frequency-dependent detection statistics for radio auroral pulsations. More lower frequency observations $(\sim 1 \mathrm{GHz})$ are required to test this hypothesis. The large overlap in the auroral $\mathrm{H} \alpha$ and radio detection statistics may also suggest that the refraction may not be very strong in the $4-8 \mathrm{GHz}$ radio band.

Additionally, Figure 15 illustrates that when the magnetic axis is misaligned, the instantaneous beam pattern on the sky changes in shape and direction. It transforms continuously from the pattern in the top panel to the pattern in the bottom panel (for each fixed polarization) and back over the course of the brown dwarf's rotational period, traversing large portions of the sky and achieving a total sky coverage comparable to the auroral oval scenario. In effect, the pattern can be considered as a result of the flux tube tied to the satellite traversing multiple L-shells and longitudes, doing a circuit with respect to the magnetic axis with the rotational period of the brown dwarf. These rotational effects do not occur if the magnetic field is well aligned with the rotational axis. Thus, for the auroral satellite scenario, the radio emission can produce periodic light curves at the brown dwarf rotation period with both single and multiple polarization peaks, depending on the relative geometry of the observer and the source. We show some example light curves demonstrating this effect for single-frequency channels in Figure 16. The different lines correspond to observers viewing the target at different inclinations, along the longitude $0^{\circ}$ line of Figure 15. These observers see narrow pulses as the beam pattern sweeps over their lines of sight during the rotational modulation of the brown dwarf. Additionally, the changes in the magnetic field strength at the location of the satellite also contribute to variability in the observed intensity of the radio pulses. Superimposed on this rotational variation, the satellite scenario further predicts a modulation of the radio emission on the orbital period of the planet around the brown dwarf. Radio emission models of TVLM 513-46546 might show some indications of this behavior (Leto et al. 2017). The long-term orbital variations may be responsible for the observed changes in pulse polarization observations, alternatively interpreted as a potential indication of stellar cycles in UCDs (Route 2016). For a single object hosting both of these electrodynamic engines, the co-rotation breakdown and a satellite flux tube, depending on the relative viewing geometry and underlying parameters, the observer might intercept both, none, or only one of the radio beam patterns of the ECMI emission. Long-term radio monitoring of these targets will provide a means to potentially measure these effects (see also Wolszczan \& Route 2014).

\subsection{Quiescent Radio Emission}

An important aspect of the detection of radio emission from UCDs has been the distinction between the pulsed emission and a quiescent component at $\mathrm{GHz}$ frequencies. While the characteristics of the pulsed emission have identified it as due to ECMI, the cause of the quiescent component is poorly understood. Based on the beam patterns and light curves from Section 3.4, there could be ECMI emission between pulse peaks, although it would likely be much weaker and variable than the idealized emissions considered here. However, the polarization of the quiescent emission does not reflect a potential ECMI origin (however, see Williams et al. 2017). UCD quiescent radio emission typically shows low levels of circular polarization; however, the constraints have not been particularly stringent (e.g., Kao et al. 2016). Based on radio detections at $\sim 100 \mathrm{GHz}$, Williams et al. (2015b) showed that the quiescent emission of TVLM 513-46546, one of the benchmark targets with periodic pulsations, was consistent with synchrotron and/or gyrosynchrotron emission. Whether the energetic electrons are highly relativistic or only mildly relativistic has been difficult to assess. Consequently, the polarization of the quiescent component could be an important distinguishing feature. A gyrosynchrotron source would show some circular polarization, whereas a synchrotron source would display significant linear polarization (Dulk 1985). The low degree of circular polarization argues against ECMI and may place constraints on gyrosynchrotron, but the future confirmation of significant linear polarization will be an important indicator of the synchrotron source for the quiescent radio component and the relativistic nature of the energetic electron population.

This, however, raises the question as to how these brown dwarfs energize the electrons responsible for this relativistic emission. Stellar gyrosynchrotron emission is connected to the same heating and acceleration mechanisms that power the hot plasma of the coronae and stellar flares. However, the lack of X-ray emission (see Figure 2) suggests that these processes are weak in the cool atmospheres of UCDs, especially in late L-dwarfs and T-dwarfs, many of which have quiescent radio detections (e.g., Kao et al. 2016). Instead, one possibility for this emission, as first proposed in Hallinan et al. (2006), is a brown dwarf counterpart to the synchrotron radiation belts of the Jovian system. Jupiter exhibits a large population of magnetospherically confined energetic electrons $(\sim 1 \mathrm{MeV})$ in its equatorial regions that generate strong synchrotron emission 


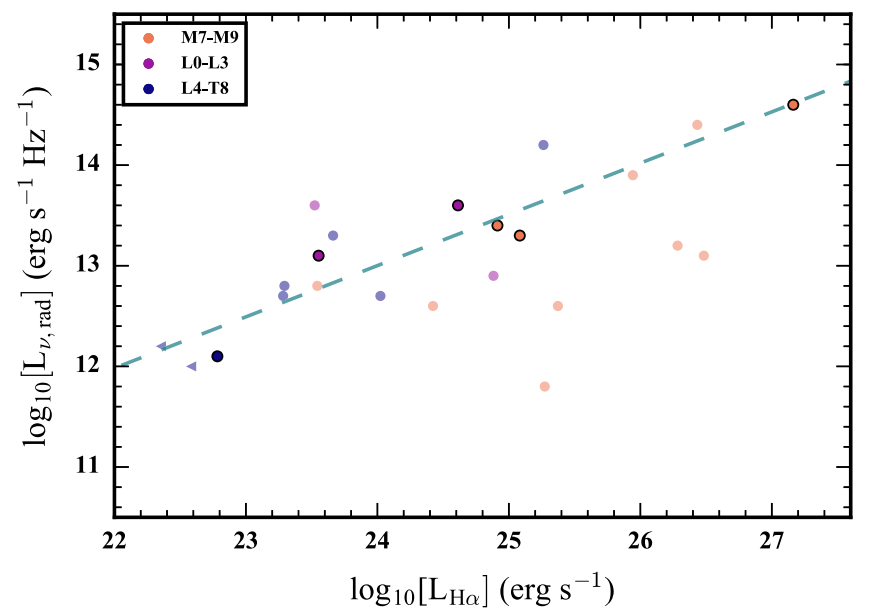

Figure 17. Observed quiescent radio luminosity of radio UCDs plotted against their $\mathrm{H} \alpha$ luminosities, taken from Tables 1 and 2 . The objects with confirmed periodic pulsations (from Table 1) are outlined in black, showing a correlation between the two emission types $(r=0.95, p=0.004)$; a best-fit regression line of slope 0.51 and ordinate intercept 0.78 for these objects is plotted as a dashed line. This suggests that there may be a connection between the auroral processes and the quiescent radio emission, see Section 3.5. The points are plotted in different shades according to their spectral types, as in Figure 1, with limits indicated by triangles.

at decimeter wavelengths (see Bagenal et al. 2014 and references therein). If similar structures exist on these brown dwarfs, they could generate the quiescent emission at $\mathrm{GHz}$ and higher frequencies, given the comparatively stronger magnetic field strength of brown dwarfs relative to Jupiter.

Although the cause of the quiescent emission remains an open question, there may be a clue to its origins in its relation to the ECMI pulsed radio emission. There is a large overlap between the population of objects with periodic pulsed ECMI emission and those with a detected quiescent radio component (e.g., Hallinan et al. 2008; Kao et al. 2016). Moreover, for many objects, initial radio detections come from short surveys of only 1-2 hr per object, which can be much less than the rotational periods, potentially missing the periodic nature of some of the bursts, or lacking sufficient sensitivity to probe quiescent emission (e.g., McLean et al. 2012; Route \& Wolszczan 2013, 2016b). Follow-up efforts on these detections often confirm the presence of periodic pulsations on top of a quiescent radio background (e.g., Route \& Wolszczan 2012; Williams \& Berger 2015). Additionally, many of the sources listed in Table 2 already show evidence for periodic pulsations, but await further follow-up observations for confirmation (Kao et al. 2016). This connection between the two kinds of radio emissions is consistent with the presence of brown dwarf synchrotron radiation belts. If this is indeed the source of the quiescent emission, then the inner magnetospheric region must be loaded with energetic plasma - a requirement similarly critical for the generation of auroral currents. In the Jovian case, the major plasma source is the moon Io (e.g., Bolton et al. 2015). The material picked up from the moon is ionized and energized by the fast rotating magnetosphere, the ultimate source of energy for the system, leading to a confined population of highly energetic electrons in the inner magnetosphere (Bolton et al. 2015). The presence of this plasma in the large-scale magnetosphere may be the crucial link connecting the processes.

Interestingly, there also appears to be a correlation between the quiescent radio emission and $\mathrm{H} \alpha$ emission strengths. In
Figure 17, we plot the quiescent radio luminosity against the $\mathrm{H} \alpha$ luminosity for radio UCDs (taken from Tables 1 and 2). These data show a Pearson correlation coefficient of 0.54 $(p=0.01),{ }^{5}$ indicating a positive correlation between the values. If we restrict the data to just the periodically pulsing sources from Table 1, the Pearson correlation coefficient rises to 0.95 ( $p=0.004$; see footnote 5). Although there are not very many data points, they further indicate a possible connection between the mechanisms producing the aurorae and the quiescent radio emission. In Figure 17, we also show the best-fit line to just the periodically pulsing sources. For confirmed ECMI objects, the brighter $\mathrm{H} \alpha$ sources correspond to the brighter quiescent radio sources. The majority of the other quiescent radio emitters loosely cluster around this bestfit line. The outliers, to the lower right, correspond preferentially to the warmer UCDs. This is likely a consequence of their $\mathrm{H} \alpha$ emission having a significant chromospheric contribution. Consequently, the warmer brown dwarfs may be systematically farther to the right in Figure 17 than is warranted by any auroral contribution to their $\mathrm{H} \alpha$ emission. Although it is unclear how this connection originates, especially considering the uncertain nature of the quiescent radio component, it is possible that both mechanisms rely not only on the same conditions that make a particular brown dwarf amenable to hosting the auroral electrodynamic engine, but both become stronger when that engine is more energetic, e.g., faster rotation, higher magnetospheric plasma densities, and stronger field strengths. Alternatively, the acceleration mechanism of the electrons responsible for the quiescent emission may be the same as that which accelerates the auroral electrons. A deeper understanding of the quiescent emission is needed to disentangle these physical effects.

\section{Conclusions}

The observational shifts in stellar magnetic activity into the UCD regime reflect the transition in physical properties in brown dwarfs going from stars to planets. The wide breadth of properties encompasses effects important in both the stellar and planetary cases. Our examination of the trends suggests that chromospheric and coronal heating begins to decay in very low-mass stars at the $M / L$ transition, where the X-ray emission drops off dramatically. Relatively weaker heating continues into the L-dwarfs, but is mostly suppressed in late L-dwarfs and $\mathrm{T}$-dwarfs following the decline in atmospheric ionization fraction. If the stark drop-off in X-rays at the $M / L$ transition signals a steep decline in significant flare heating, it is possible that the residual chromospheres of L-dwarfs may be sustained by a continued gradual decline in MHD wave dissipation with cooler atmospheric temperatures. A better understanding of the flare frequency distribution in the UCD regime will help elucidate the nature of this transition.

Amidst this transition, some brown dwarf systems exhibit the conditions required to power an auroral electrodynamic engine. We stress that aurorae, as we have defined in this article, do not require an external star, but can be generated internally by the brown dwarf system, as a consequence of large-scale magnetospheric currents. Although the exact conditions are unclear, they require strong magnetic field

\footnotetext{
5 The data sample is small and thus the $p$ values associated with the Pearson correlation coefficient, $r$, are not particularly meaningful, but are included here for completeness.
} 
strengths, large-scale magnetic field topologies, fast rotation rates, and the presence of significant magnetospheric plasma. Although there are other possibilities (see Section 3), the last condition is possibly associated with the presence of planets around brown dwarfs, analogous to the Jupiter-Io system, which seeds the magnetosphere with plasma through the moon's volcanic activity. Although the first three requirements are potentially met by most, if not all, brown dwarfs, the origins of the plasma and the role of a planetary satellite could be the underlying feature that distinguishes aurorally active brown dwarfs from inactive ones. This could potentially tie the auroral detection statistics to the planet formation rates around brown dwarfs and/or to the presence of particular satellite system architectures.

The ECMI radio emission, periodically pulsed, coherent, and with high degrees of circular polarization, is the key observational indicator of the existence of auroral magnetospheric processes. The aurorae are defined fundamentally by the presence of strong-field-aligned currents and a precipitating electron beam impacting the atmosphere. The consequences of this auroral beam include multiwavelength surface emission features, like $\mathrm{H} \alpha$ and $\mathrm{H}_{2}$ Werner band emission in addition to the pulsed radio emission. Furthermore, the various multiwavelength emissions provide significant probes of the brown dwarf atmospheres: temperature, ionization, chemistry. Moreover, these emissions may provide the only probes of brown dwarf upper atmospheres and could provide constraints on the properties of the energetic electron distribution and strength of the auroral currents. Because the atmospheric and electrodynamic conditions deviate considerably from the Jovian example, the expected energy balance of the different processes could be very different; however, more observations are required to establish the breakdown of total auroral energy dissipation. Furthermore, auroral brown dwarfs provide an opportunity to explore a new parameter space in auroral physics, relative to what is seen in the solar system: stronger magnetic field strengths, faster rotation rates, and warmer and denser atmospheres.

Understanding these effects properly will require further investigation in both observations of brown dwarfs and modeling the impact of these processes in their atmospheres. Much remains uncertain but, like the Sun and stellar activity, Jupiter and auroral activity will continue to provide important clues in deciphering the physics underlying brown dwarf magnetism at the cross-section of stars and planets.

\section{Summary}

In this article, we discussed how the trends in magnetic activity shift in the UCD regime and what the implications are for the underlying mechanisms powering magnetic phenomena in brown dwarf atmospheres, and in particular how auroral phenomena fit into these observations. Moreover, we applied the auroral paradigm to the multiwavelength features of brown dwarf emission, illustrating the various processes and observational signatures indicative of UCD aurorae.

We summarize our main findings below:

1. The distributions of $\mathrm{H} \alpha$ emission in the UCD regime show a transition across L spectral types from predominantly coronal/chromospheric to likely auroral.

2. The strength of X-ray and $\mathrm{H} \alpha$ emissions in M7-M9 dwarfs are correlated over two orders of magnitude in $\mathrm{X}$-ray luminosity.
3. The predominant electrodynamic engine of auroral brown dwarfs may be co-rotation breakdown, and could involve the presence of close-in planetary companions to the brown dwarfs.

4. The auroral power is closely tied to the magnetic field strength and rotational velocity of the brown dwarf, regardless of the underlying engine.

5. The presence of auroral emissions should be correlated with the presence of large-scale dipolar magnetic field topologies.

6. Brown dwarf auroral atmospheric emissions, such as $\mathrm{H} \alpha$, $\mathrm{H}_{3}^{+}$, and $\mathrm{H}_{2}$ Werner band emission, are likely generated in auroral surface features but may be affected by atmospheric conditions.

7. The auroral surface feature morphology is imprinted on the shape of the emission lines and depends on the electrodynamic engine powering the currents creating the auroral feature.

8. Large-amplitude NIR variability is likely dominated by transient cloud features and not magnetic effects, although the influence of auroral activity may be responsible for the long-lived sinusoidal features observed in photometric monitoring of some objects.

9. ECMI radio beaming patterns can produce a broad variety of observed light curves, accounting for the different morphologies of radio emissions from brown dwarfs hosting highly polarized periodic radio pulses.

10. The observed radio variability can be strongly dependent on the relative geometry of the source and the observer.

11. The potentially low degree of geometric selection effect in the observed detections of radio pulses suggests that brown dwarf magnetic axes may be significantly misaligned in general.

12. Most quiescent radio sources with extended monitoring observations have also been detected as sources of periodic, highly circularly polarized radio emission.

13. Quiescent radio luminosities are correlated with $\mathrm{H} \alpha$ luminosities for confirmed periodically pulsing UCDs, suggesting a physical connection between the quiescent radio emission and the conditions generating brown dwarf auroral emission.

J.S.P. was supported by a grant from the National Science Foundation Graduate Research Fellowship under grant No. (DGE-11444469). J.S.P. would like to thank Jackie Villadsen for useful discussions in the development of the arguments presented in this article. The authors would also like to thank the anonymous referee for a thorough reading of this manuscript and for providing critical feedback that greatly strengthened this work.

This research has benefited from the M-, L-, T-, and Y-dwarf compendium housed at DwarfArchives.org. This research has benefited from the Ultracool RIZzo Spectral Library maintained by Jonathan Gagné and Kelle Cruz. This researched has benefited from the Database of Ultracool Parallaxes maintained by Trent Dupuy.

This publication makes use of data products from the Two Micron All Sky Survey, which is a joint project of the University of Massachusetts and the Infrared Processing and Analysis Center/California Institute of Technology, funded by the National Aeronautics and Space Administration and the National Science Foundation. 


\section{Appendix}

Throughout this paper, we have compiled the results of many literature sources measuring the different properties of UCDs. Below, we report the sources for the data used in this compilation, according to the measurement referenced. In many cases, there have been multiple observations of the same properties for individual stars. In this article, we use the values that are more recent and illustrate consistency between the reports of multiple groups.

\section{Projected Rotational Velocities}

Mohanty \& Basri (2003), Mohanty et al. (2003), Blake et al. (2010), Tanner et al. (2012), Prato et al. (2015), Gizis et al. (2016), and Crossfield (2014, and references therein).

\section{$H \alpha$}

Kirkpatrick et al. (1999, 2000), Gizis et al. (2000, 2013), Hall (2002), Mohanty \& Basri (2003), Burgasser et al. (2003, 2011, 2015b), Liebert et al. (2003), Reiners \& Basri (2007, 2008), Schmidt et al. (2007), Lee et al. (2010), West et al. (2011), and Metodieva et al. (2015), Pineda et al. (2016).

\section{Radio}

Berger (2002, 2006), Berger et al. (2005, 2009, 2010), Burgasser et al. (2015c), Burgasser \& Putman (2005), Osten \& Jayawardhana (2006), Hallinan et al. (2007, 2008), McLean et al. (2012), Route \& Wolszczan (2012, 2013, 2016a, 2016b), Antonova et al. (2013), Gizis et al. (2013), Osten et al. (2015), Williams \& Berger (2015), Williams et al. (2015b), Kao et al. (2016), and Lynch et al. (2016).

\section{$X$-Ray}

Tsuboi et al. (2003), Gizis \& Bharat (2004), Berger et al. (2005, 2010), Audard et al. (2005), Osten et al. (2015), Cook et al. (2014), and Williams et al. (2014, and references therein).

\section{ORCID iDs}

J. Sebastian Pineda (iఠ https://orcid.org/0000-0002-4489-0135 Melodie M. Kao (1) https://orcid.org/0000-0001-5125-1414

\section{References}

Alibert, Y., \& Benz, W. 2017, A\&A, 598, L5

Antonova, A., Hallinan, G., Doyle, J. G., et al. 2013, A\&A, 549, A131 Apai, D., Radigan, J., Buenzli, E., et al. 2013, ApJ, 768, 121

Artigau, É., Bouchard, S., Doyon, R., \& Lafreniere, D. 2009, ApJ, 701, 1534 Artigau, É., Doyon, R., Lafrenière, D., et al. 2006, ApJL, 651, L57

Audard, M., Brown, A., Briggs, K. R., et al. 2005, ApJL, 625, L63

Badman, S. V., Branduardi-Raymont, G., Galand, M., et al. 2015, SSRv, 187, 99

Bagenal, F., Adriani, A., Allegrini, F., et al. 2014, SSRv, 4

Barnes, J. R., Jeffers, S. V., Jones, H. R. A., et al. 2015, ApJ, 812, 42

Benz, A. O., \& Guedel, M. 1994, A\&A, 285, 621

Berger, E. 2002, ApJ, 572, 503

Berger, E. 2006, ApJ, 648, 629

Berger, E., Ball, S., Becker, K. M., et al. 2001, Natur, 410, 338

Berger, E., Basri, G., Fleming, T. A., et al. 2010, ApJ, 709, 332

Berger, E., Basri, G., Gizis, J. E., et al. 2008, ApJ, 676, 1307

Berger, E., Rutledge, R. E., Phan-Bao, N., et al. 2009, ApJ, 695, 310

Berger, E., Rutledge, R. E., Reid, I. N., et al. 2005, ApJ, 627, 960

Bessell, M. S. 1991, AJ, 101, 662

Bhardwaj, A., \& Gladstone, G. R. 2000, RvGeo, 38, 295
Biller, B. A., Crossfield, I. J. M., Mancini, L., et al. 2013, ApJL, 778, L10

Blake, C. H., Charbonneau, D., \& White, R. J. 2010, ApJ, 723, 684

Bolton, S. J., Bagenal, F., Blanc, M., et al. 2015, SSRv, 192, 209

Bouy, H., Duchêne, G., Köhler, R., et al. 2004, A\&A, 423, 341

Browning, M. K. 2008, ApJ, 676, 1262

Browning, M. K., Basri, G., Marcy, G. W., West, A. A., \& Zhang, J. 2010, AJ, 139,504

Browning, M. K., Miesch, M. S., Brun, A. S., \& Toomre, J. 2006, ApJL, 648, L157

Buenzli, E., Apai, D., Radigan, J., Reid, I. N., \& Flateau, D. 2014, ApJ, 782, 77 Buenzli, E., Saumon, D., Marley, M. S., et al. 2015, ApJ, 798, 127

Burgasser, A. J., Geballe, T. R., Leggett, S. K., Kirkpatrick, J. D., \& Golimowski, D. A. 2006, ApJ, 637, 1067

Burgasser, A. J., Gillon, M., Melis, C., et al. 2015a, AJ, 149, 104

Burgasser, A. J., Kirkpatrick, J. D., Liebert, J., \& Burrows, A. 2003, ApJ, 594, 510

Burgasser, A. J., Logsdon, S. E., Gagne, J., et al. 2015b, ApJS, 220, 18 Burgasser, A. J., Melis, C., Todd, J., et al. 2015c, AJ, 150, 180

Burgasser, A. J., Melis, C., Zauderer, B. A., \& Berger, E. 2013, ApJL, 762, L3 Burgasser, A. J., \& Putman, M. E. 2005, ApJ, 626, 486

Burgasser, A. J., Reid, I. N., Leggett, S. K., et al. 2005, ApJL, 634, L177

Burgasser, A. J., Sitarski, B. N., Gelino, C. R., Logsdon, S. E., \& Perrin, M. D. 2011, ApJ, 739, 49

Burrows, A., Hubbard, W. B., Lunine, J. I., \& Liebert, J. 2001, RvMP, 73, 719

Castro, P. J., Gizis, J. E., Harris, H. C., et al. 2013, ApJ, 776, 126

Chabrier, G., \& Baraffe, I. 2000, ARA\&A, 38, 337

Christensen, U. R., Holzwarth, V., \& Reiners, A. 2009, Natur, 457, 167

Clarke, F. J., Hodgkin, S. T., Oppenheimer, B. R., Robertson, J., \& Haubois, X. 2008, MNRAS, 386, 2009

Cook, B. A., Williams, P. K. G., \& Berger, E. 2014, ApJ, 785, 10

Covey, K. R., Agüeros, M. A., Lemonias, J. J., et al. 2011, in ASP Conf. Ser. 448, XVI Cambridge Workshop on Cool Stars, Stellar Systems, and the Sun, ed. C. Johns-Krull, M. K. Browning, \& A. A. West (San Francisco, CA: ASP), 269

Cowley, S., Bunce, E., \& Prangé, R. 2004, AnGeo, 22, 1379

Cowley, S. W. H., \& Bunce, E. J. 2001, P\&SS, 49, 1067

Cram, L. E., \& Mullan, D. J. 1985, ApJ, 294, 626

Croll, B., Muirhead, P. S., Han, E., et al. 2016a, arXiv:1609.03586

Croll, B., Muirhead, P. S., Lichtman, J., et al. 2016b, arXiv:1609.03587

Crossfield, I. J. M. 2014, A\&A, 566, A130

Crossfield, I. J. M., Biller, B., Schlieder, J. E., et al. 2014, Natur, 505, 654

Cruz, K. L., Reid, I. N., Kirkpatrick, J. D., et al. 2007, AJ, 133, 439

Cruz, K. L., Reid, I. N., Liebert, J., Kirkpatrick, J. D., \& Lowrance, P. J. 2003, AJ, 126, 2421

Delfosse, X., Forveille, T., Perrier, C., \& Mayor, M. 1998, A\&A, 331, 581

Donati, J.-F., Forveille, T., Collier Cameron, A., et al. 2006, Sci, 311, 633

Donati, J.-F., Morin, J., Petit, P., et al. 2008, MNRAS, 390, 545

Dulk, G. A. 1985, ARA\&A, 23, 169

Dupuy, T. J., Forbrich, J., Rizzuto, A., et al. 2016, ApJ, 827, 23

Dupuy, T. J., Liu, M. C., Bowler, B. P., et al. 2010, ApJ, 721, 1725

Dyudina, U. A., Ingersoll, A. P., Ewald, S. P., \& Wellington, D. 2016, Icar, 263,32

Enoch, M. L., Brown, M. E., \& Burgasser, A. J. 2003, AJ, 126, 1006

Filippazzo, J. C., Rice, E. L., Faherty, J., et al. 2015, ApJ, 810, 158

Forbrich, J., Dupuy, T. J., Reid, M. J., et al. 2016, ApJ, 827, 22

Forveille, T., Beuzit, J.-L., Delorme, P., et al. 2005, A\&A, 435, L5

France, K., Froning, C. S., Linsky, J. L., et al. 2013, ApJ, 763, 149

France, K., Parke Loyd, R. O., Youngblood, A., et al. 2016, ApJ, 820, 89

Freed, M., Close, L. M., \& Siegler, N. 2003, ApJ, 584, 453

Gillon, M., Jehin, E., Lederer, S. M., et al. 2016, Natur, 533, 221

Gillon, M., Jehin, E., Magain, P., et al. 2011, EPJ Web of Conf. 11, Detection and Dynamics of Transiting Exoplanets, ed. F. Bouchy, R. Díaz, \& C. Moutou (Les Ulis: EDP Sciences), 06002

Gillon, M., Triaud, A. H. M. J., Demory, B.-O., et al. 2017, Natur, 542, 456

Gizis, J. E. 2002, ApJ, 575, 484

Gizis, J. E., \& Bharat, R. 2004, ApJL, 608, L113

Gizis, J. E., Burgasser, A. J., Berger, E., et al. 2013, ApJ, 779, 172

Gizis, J. E., Dettman, K. G., Burgasser, A. J., et al. 2015, ApJ, 813, 104

Gizis, J. E., Monet, D. G., Reid, I. N., et al. 2000, AJ, 120, 1085

Gizis, J. E., Paudel, R. R., Schmidt, S. J., Williams, P. K. G., \& Burgasser, A. J. 2017, ApJ, 838, 22

Gizis, J. E., Troup, N. W., \& Burgasser, A. J. 2011, ApJL, 736, L34

Gizis, J. E., Williams, P. K. G., Burgasser, A. J., et al. 2016, AJ, 152, 123

Gladstone, G. R., Waite, J. H., Grodent, D., et al. 2002, Natur, 415, 1000

Grodent, D., Clarke, J. T., Kim, J., Waite, J. H., \& Cowley, S. W. H. 2003, JGRA, 108, 1389 
Güdel, M. 2002, ARA\&A, 40, 217

Guedel, M., \& Benz, A. O. 1993, ApJL, 405, L63

Gustin, J., Gérard, J.-C., Grodent, D., et al. 2013, JMoSp, 291, 108

Haisch, B., Strong, K. T., \& Rodono, M. 1991, ARA\&A, 29, 275

Hall, P. B. 2002, ApJL, 564, L89

Hallinan, G., Antonova, A., Doyle, J. G., et al. 2006, ApJ, 653, 690

Hallinan, G., Antonova, A., \& Doyle, J. G. 2008, ApJ, 684, 644

Hallinan, G., Bourke, S., Lane, C., et al. 2007, ApJL, 663, L25

Hallinan, G., Littlefair, S. P., Cotter, G., et al. 2015, Natur, 523, 568

Harding, L. K., Hallinan, G., Boyle, R. P., et al. 2013a, ApJ, 779, 101

Harding, L. K., Hallinan, G., Konopacky, Q. M., et al. 2013b, A\&A, 554, A113

Hawley, S. L., \& Johns-Krull, C. M. 2003, ApJL, 588, L109

He, M. Y., Triaud, A. H. M. J., \& Gillon, M. 2017, MNRAS, 464, 2687

Heinze, A. N., Metchev, S., \& Kellogg, K. 2015, ApJ, 801, 104

Hess, S. L. G., Bonfond, B., Zarka, P., \& Grodent, D. 2011, JGRA, 116, A05217

Hilton, E. J., West, A. A., Hawley, S. L., \& Kowalski, A. F. 2010, AJ, 140,1402

Hui, Y., Schultz, D. R., Kharchenko, V. A., et al. 2009, ApJL, 702, L158

Irwin, J., \& Bouvier, J. 2009, in IAU Symp. 258, The Ages of Stars, ed.

E. E Mamajek, D. R. Soderblom, \& R. F. G. Wyse (Cambridge: Cambridge Univ. Press), 363

James, D. J., Jardine, M. M., Jeffries, R. D., et al. 2000, MNRAS, 318, 1217 Jones, D. O., \& West, A. A. 2016, ApJ, 817, 1

Kao, M. M., Hallinan, G., Pineda, J. S., et al. 2016, ApJ, 818, 24

Keiling, A., Donovan, E., Bagenal, F., \& Karlsson, T. 2012, GMS, 197

Khandrika, H., Burgasser, A. J., Melis, C., et al. 2013, AJ, 145, 71

Kiraga, M., \& Stepien, K. 2007, AcA, 57, 149

Kirkpatrick, J. D., Cushing, M. C., Gelino, C. R., et al. 2011, ApJS, 197, 19

Kirkpatrick, J. D., Henry, T. J., \& Simons, D. A. 1995, AJ, 109, 797

Kirkpatrick, J. D., Reid, I. N., Liebert, J., et al. 1999, ApJ, 519, 802

Kirkpatrick, J. D., Reid, I. N., Liebert, J., et al. 2000, AJ, 120, 447

Kitchatinov, L. L., Moss, D., \& Sokoloff, D. 2014, MNRAS, 442, L1

Koen, C. 2013, MNRAS, 428, 2824

Koenker, R. 2016, quantreg: Quantile Regression, $r$ package version 5.24

Konopacky, Q. M., Ghez, A. M., Barman, T. S., et al. 2010, ApJ, 711, 1087

Lee, K.-G., Berger, E., \& Knapp, G. R. 2010, ApJ, 708, 1482

Leggett, S. K., Allard, F., Geballe, T. R., Hauschildt, P. H., \& Schweitzer, A. 2001, ApJ, 548, 908

Leto, P., Trigilio, C., Buemi, C. S., et al. 2016, MNRAS, 459, 1159

Leto, P., Trigilio, C., Buemi, C. S., et al. 2017, MNRAS, 469, 1949

Liebert, J., Kirkpatrick, J. D., Cruz, K. L., et al. 2003, AJ, 125, 343

Liebert, J., Kirkpatrick, J. D., Reid, I. N., \& Fisher, M. D. 1999, ApJ, 519, 345

Linsky, J. L. 1980, ARA\&A, 18, 439

Lynch, C., Murphy, T., Ravi, V., et al. 2016, MNRAS, 457, 1224

Lynch, C., Mutel, R. L., \& Güdel, M. 2015, ApJ, 802, 106

Mahadevan, S., Ramsey, L., Bender, C., et al. 2012, Proc. SPIE, 8446, 84461S

Maillard, J., \& Miller, S. 2011, in ASP Conf. Ser. 450, Molecules in the Atmospheres of Extrasolar Planets, ed. J. P. Beaulieu, S. Dieters, \& G. Tinetti (San Francisco, CA: ASP), 19

Marley, M. S., \& Robinson, T. D. 2015, ARA\&A, 53, 279

McLean, M., Berger, E., Irwin, J., Forbrich, J., \& Reiners, A. 2011, ApJ, 741,27

McLean, M., Berger, E., \& Reiners, A. 2012, ApJ, 746, 23

Metchev, S. A., Heinze, A., Apai, D., et al. 2015, ApJ, 799, 154

Metodieva, Y., Antonova, A., Golev, V., et al. 2015, MNRAS, 446, 3878

Miles-Páez, P. A., Metchev, S. A., Heinze, A., \& Apai, D. 2017, ApJ, 840, 83

Mohanty, S., \& Basri, G. 2003, ApJ, 583, 451

Mohanty, S., Basri, G., Shu, F., Allard, F., \& Chabrier, G. 2002, ApJ, 571, 469

Mohanty, S., Jayawardhana, R., \& Barrado y Navascués, D. 2003, ApJL, 593, L109

Morin, J., Donati, J.-F., Petit, P., et al. 2010, MNRAS, 407, 2269

Morin, J., Dormy, E., Schrinner, M., \& Donati, J.-F. 2011, MNRAS, 418, L133

Morley, C. V., Marley, M. S., Fortney, J. J., \& Lupu, R. 2014, ApJL, 789, L14

Mutel, R. L., Christopher, I. W., \& Pickett, J. S. 2008, GeoRL, 35, L07104

Newton, E. R., Irwin, J., Charbonneau, D., et al. 2017, ApJ, 834, 85

Nichols, J. D., Burleigh, M. R., Casewell, S. L., et al. 2012, ApJ, 760, 59

Nutzman, P., \& Charbonneau, D. 2008, PASP, 120, 317

Ossendrijver, M. 2003, A\&ARv, 11, 287

Osten, R. A., \& Jayawardhana, R. 2006, ApJL, 644, L67

Osten, R. A., Melis, C., Stelzer, B., et al. 2015, ApJL, 805, L3

Peale, S. J. 1999, ARA\&A, 37, 533
Perry, J. J., Kim, Y. H., Fox, J. L., \& Porter, H. S. 1999, JGR, 104, 16541

Pineda, J. S., Hallinan, G., Kirkpatrick, J. D., et al. 2016, ApJ, 826, 73

Pineda, J. S., West, A. A., Bochanski, J. J., \& Burgasser, A. J. 2013, AJ, 146,50

Pizzolato, N., Maggio, A., Micela, G., Sciortino, S., \& Ventura, P. 2003, A\&A, 397, 147

Prato, L., Mace, G. N., Rice, E. L., et al. 2015, ApJ, 808, 12

R Core Team 2016, R: A Language and Environment for Statistical Computing, R Foundation for Statistical Computing, Vienna, Austria, https://www.R-project.org/

Radigan, J., Jayawardhana, R., Lafrenière, D., et al. 2012, ApJ, 750, 105

Radigan, J., Lafrenière, D., Jayawardhana, R., \& Artigau, E. 2014, ApJ, 793, 75

Reid, I. N., Cruz, K. L., Kirkpatrick, J. D., et al. 2008, AJ, 136, 1290

Reid, I. N., Cruz, K. L., Laurie, S. P., et al. 2003, AJ, 125, 354

Reid, I. N., Kirkpatrick, J. D., Liebert, J., et al. 2002, AJ, 124, 519

Reiners, A., \& Basri, G. 2007, ApJ, 656, 1121

Reiners, A., \& Basri, G. 2008, ApJ, 684, 1390

Reiners, A., \& Basri, G. 2010, ApJ, 710, 924

Reiners, A., \& Mohanty, S. 2012, ApJ, 746, 43

Ribas, I., Bolmont, E., Selsis, F., et al. 2016, A\&A, 596, A111

Ricker, G. R., Winn, J. N., Vanderspek, R., et al. 2014, Proc. SPIE, 9143, 914320

Robinson, T. D., \& Marley, M. S. 2014, ApJ, 785, 158

Rodriguez-Barrera, M. I., Helling, C., Stark, C. R., \& Rice, A. M. 2015, MNRAS, 454, 3977

Rosner, R., Golub, L., \& Vaiana, G. S. 1985, ARA\&A, 23, 413

Route, M. 2016, ApJL, 830, L27

Route, M., \& Wolszczan, A. 2012, ApJL, 747, L22

Route, M., \& Wolszczan, A. 2013, ApJ, 773, 18

Route, M., \& Wolszczan, A. 2016a, ApJL, 821, L21

Route, M., \& Wolszczan, A. 2016b, ApJ, 830, 85

Saur, J., Neubauer, F. M., Connerney, J. E. P., Zarka, P., \& Kivelson, M. G. 2004, in Jupiter. The planet, satellites and magnetosphere, ed. F. Bagenal, T. E. Dowling, \& W. B. McKinnon (Cambridge: Cambridge Univ. Press), 537

Schmidt, S. J., Cruz, K. L., Bongiorno, B. J., Liebert, J., \& Reid, I. N. 2007, AJ, 133, 2258

Schmidt, S. J., Hawley, S. L., West, A. A., et al. 2015, AJ, 149, 158

Schmidt, S. J., Prieto, J. L., Stanek, K. Z., et al. 2014a, ApJL, 781, L24

Schmidt, S. J., West, A. A., Bochanski, J. J., Hawley, S. L., \& Kielty, C. 2014b, PASP, 126, 642

Schrijver, C. J. 2009, ApJL, 699, L148

Shkolnik, E. L., \& Barman, T. S. 2014, AJ, 148, 64

Siegler, N., Close, L. M., Cruz, K. L., Martín, E. L., \& Reid, I. N. 2005, ApJ, 621,1023

Skumanich, A. 1972, ApJ, 171, 565

Solanki, S. K., Inhester, B., \& Schüssler, M. 2006, RPPh, 69, 563

Stelzer, B., Alcalá, J., Biazzo, K., et al. 2012, A\&A, 537, A94

Tanner, A., White, R., Bailey, J., et al. 2012, ApJS, 203, 10

Tao, C., Badman, S. V., \& Fujimoto, M. 2011, Icar, 213, 581

Tao, C., Badman, S. V., Uno, T., \& Fujimoto, M. 2012, Icar, 221, 236

Treumann, R. A. 2006, A\&ARv, 13, 229

Tsuboi, Y., Maeda, Y., Feigelson, E. D., et al. 2003, ApJL, 587, L51

Udalski, A., Jung, Y. K., Han, C., et al. 2015, ApJ, 812, 47

Vasavada, A. R., Bouchez, A. H., Ingersoll, A. P., et al. 1999, JGR, 104, 27133

Walkowicz, L. M., \& Hawley, S. L. 2009, AJ, 137, 3297

West, A. A., Hawley, S. L., Bochanski, J. J., et al. 2008, AJ, 135, 785

West, A. A., Morgan, D. P., Bochanski, J. J., et al. 2011, AJ, 141, 97

West, A. A., Weisenburger, K. L., Irwin, J., et al. 2015, ApJ, 812, 3

Williams, P. K. G., \& Berger, E. 2015, ApJ, 808, 189

Williams, P. K. G., Berger, E., Irwin, J., Berta-Thompson, Z. K., \& Charbonneau, D. 2015a, ApJ, 799, 192

Williams, P. K. G., Casewell, S. L., Stark, C. R., et al. 2015b, ApJ, 815, 64

Williams, P. K. G., Cook, B. A., \& Berger, E. 2014, ApJ, 785, 9

Williams, P. K. G., Gizis, J. E., \& Berger, E. 2017, ApJ, 834, 117

Wilson, P. A., Rajan, A., \& Rajan, A. 2014, A\&A, 566, A111

Wolszczan, A., \& Route, M. 2014, ApJ, 788, 23

Wright, N. J., \& Drake, J. J. 2016, Natur, 535, 526

Yadav, R. K., Christensen, U. R., Morin, J., et al. 2015, ApJL, 813, L31

Yu, S., Hallinan, G., Doyle, J. G., et al. 2011, A\&A, 525, A39

Zarka, P. 1998, JGR, 103, 20159

Zarka, P. 2007, P\&SS, 55, 598 